\title{
Covid-19 pandemic and the unprecedented mobilisation of scholarly efforts prompted by a health crisis: Scientometric comparisons across SARS, MERS and 2019-nCoV literature
}

\author{
Milad Haghani ${ }^{1} \cdot$ Michiel C. J. Bliemer $^{1}$
}

Received: 1 June 2020 / Accepted: 1 September 2020 / Published online: 21 September 2020

(c) Akadémiai Kiadó, Budapest, Hungary 2020

\begin{abstract}
During the current century, each major coronavirus outbreak has triggered a quick and immediate surge of academic publications on its respective topic. The spike in research publications following the 2019 Novel Coronavirus (Covid-19) outbreak, however, has been like no other. The global crisis caused by the Covid-19 pandemic has mobilised scientific efforts at an unprecedented scale. In less than 5 months, more than 12,000 research items and in less than seven months, more than 30,000 items were indexed, while it is projected that the number could exceed 80,000 by the end of 2020 , should the current trend continues. With the health crisis affecting all aspects of life, research on Covid-19 seems to have become a focal point of interest across many academic disciplines. Here, scientometric aspects of the Covid-19 literature are analysed and contrasted with those of the two previous major coronavirus diseases, i.e., Severe Acute Respiratory Syndrome (SARS) and Middle East Respiratory Syndrome (MERS). The focus is on the co-occurrence of keyterms, bibliographic coupling and citation relations of journals and collaborations between countries. Interesting recurring patterns across all three literatures were discovered. All three outbreaks have commonly generated three distinct cohorts of studies: (i) studies linked to public health response and epidemic control, (ii) studies on chemical constitution of the virus; and (iii) studies related to treatment, vaccine and clinical care. While studies affiliated with category (i) seem to have been relatively earliest to emerge, they have overall received relatively smaller number of citations compared to publications the two other categories. Covid-19 studies seem to have been disseminated across a broader variety of journals and across a more diverse range of subject areas. Clear links are observed between the geographical origins of each outbreak as well as the local geographical severity of each outbreak and the magnitude of research originated from regions. Covid-19 studies also display the involvement of authors from a broader variety of countries compared to SARS and MERS. Considering the speed at which the Covid-19-related literature is accumulating, an interesting dimension that warrants further exploration could be to assess if the quality and rigour of these publications have been affected.
\end{abstract}

Keywords Scientometrics · Bibliometrics · Research synthesis · Coronaviruses · Viral respiratory diseases

Extended author information available on the last page of the article 


\section{Introduction}

On December 31, 2019 an official case of a novel respiratory disease of the category of coronaviruses, named Covid-19, was reported in Wuhan, China, marking the beginning of what proved to be one of the direst and most devastating viral outbreaks in the modern history (Sohrabi et al. 2020; Wang et al. 2020). This was immediately followed by an unprecedented and swift response of the academic community to address various dimensions of this health crisis and prompted an avalanche of scholarly publications on this topic (Golinelli et al. 2020; Haghani et al. 2020; Kagan et al. 2020). In less than five months, more than 12,000 publications on this topic have already been indexed by Scopus with the number increasing figuratively every day in considerable increments ${ }^{1}$ (Torres-Salinas et al. 2020). And this figure does not even include many more publications available in various repositories, including CORD-19 (Colavizza et al. 2020), in the form of preprints awaiting peer review by their respective journals. Such explosion of research on a single topic and the off-the-charts surge in the rate of publications is arguably an unprecedented trend in the history of scholarly publications. An article published by Science on May 13, 2020 , referred to this phenomenon as one that is "among the biggest explosions of scientific literature ever" (Brainard 2020). It highlighted how, in the face of this phenomenon, it has become extremely challenging for scientists to stay abreast of the latest developments. This has made the importance of research synthesis more tangible than ever and has even resulted in the development of several computational research mining tools for this very topic utilising methods such as Artificial Intelligence (AI). Among such efforts is a research synthesis tool powered by AI algorithms which has harvested datapoints from a large number the CORD-19 articles and categorised them (Brainard 2020).

Though the impact of the Covid-19 health crisis has marked it as a rather unique milestone in the history of disease outbreaks, the world, prior to this, was not a stranger with coronavirus disease outbreaks (McIntosh 1974; Myint 1994; Cavanagh 2005; Lim et al. 2016; Chen et al. 2020). Prior to 2020, two major outbreaks of this family of viruses had already been reported with at least one of them carrying the official label of a "global pandemic" (Wang et al. 2020). On November 16, 2002, the first case of the Severe Acute Respiratory Syndrome (SARS) disease was reported in the Guangdong province in southern China, which by 2003, swiftly spread from continent to continent, prompting the World Health Organisation to declare it as a pandemic. In fact, SARS is known to be "the First Pandemic of the 21 st Century" (Cherry and Krogstad 2004). Nearly ten years later, on June 13, 2012, the first case of the Middle Eastern Respiratory Syndrome (MERS) disease was discovered in Jeddah, Saudi Arabia. These two constituted the two most major coronavirus outbreaks, until Covid-19 came along. Similar to Covid-19, though at a much smaller scale, each of these previous outbreaks generated a literature of their own (Kostoff and Morse 2011).

In the face of the flood of scholarly outputs on Covid-19, and along with the conventional review and research synthesis studies (Chang et al. 2020; Chen et al. 2020; Cortegiani et al. 2020), scientometric (Colavizza et al. 2020) and bibliometric methods (BonillaAldana et al. 2020; Hossain 2020) have also gained traction in documenting and analysing the rapid developments of this literature and discovering emerging patterns (Chahrour et al. 2020; Dehghanbanadaki et al. 2020; Haghani et al. 2020; Kumar 2020; Le Bras et al.

\footnotetext{
${ }^{1}$ At the time of drafting the revision, July 21, 2020, the number had reached 30,400 according to Scopus.
} 
2020). Here in this work, the literatures of these three major coronavirus diseases are disentangled from one another and analysed in a comparative way and from scientometric perspectives. The aim is to discover possible similarities and discrepancies across these three segments of the coronavirus literature, and to discover whether there are recurring patterns in terms of magnitude, temporal evolution and the structure of these three literatures that were each developed in response to a respiratory disease outbreak. The main focus of the analyses is on keyword co-occurrences, bibliographic coupling and citation relations of sources and collaborations between countries.

\section{Methods, data and general statistics}

To compare the scientometric aspects of the studies on SARS, MERS and Covid-19, three separate datasets of publications on these three topics were retrieved from Scopus through three separate search strategies. The decision on which general database to use (e.g. Web of Science (WoS) or Scopus) was mainly made on the basis of the number of indexed Covid19 studies in particular, as the sector of the coronavirus literature that is currently emerging (compared to the literatures on SARS and MERS that have already stabilised). At the time of the data retrieval (May 2020), WoS had indexed slightly less than 5,000 research items on Covid-19, while the number of items in Scopus neared 12,000. Given the fact that the Scopus database was considerably more up to date in that respect, this database was set as the main source of data extraction in this work. Therefore, for the sake of consistency, the data for SARS and MERS were also extracted from Scopus.

The search strategies were devised in a way to minimise the possible overlap between the datasets on SARS, MERS and Covid-19 and to disentangle the three datasets from one another to the most possible extent. Preliminary inspection of the literature on each three topics determined a set of distinct keywords that would return the target literature with reasonable specificity and sensitivity. In each search, key terms associated with the other literatures were combined with the Boolean operator "AND NOT" in order to avoid the overlap. The lower bound of the time span for each search was set with consideration of the year when each viral outbreak took place. The query strings associated with the three datasets are as below:

SARS: ( TITLE-ABS-KEY ( ( ( "Severe acute respiratory syndrome" OR "SARS") AND ( coronavirus*)) OR ( "SARS virus" OR "SARS disease" OR "Severe acute respiratory syndrome disease" OR "Severe acute respiratory syndrome virus" OR "SARS-Cov")) AND NOT TITLE-ABS-KEY ( ( "covid" OR "nCov" OR "Covid-19" OR "covid19" OR "SARSCov-2" OR "Severe acute respiratory syndrome-2" OR "MERS" OR "middle east respiratory syndrome"))) AND PUBYEAR > 2001.

MERS: ( TITLE-ABS-KEY ( ( ("Middle east respiratory syndrome" OR "MERS") AND ( coronavirus)) OR ("MERS-Cov" OR "MERS virus" OR "MERS disease" OR "Middle east respiratory syndrome virus" OR "Middle east respiratory syndrome disease")) AND NOT TITLE-ABS-KEY ( ( "nCov" OR "Covid-19" OR "covid19" OR "SARS-Cov" OR "SARSCov-2" OR "SARS" OR "Severe acute respiratory syndrome"))) AND PUBYEAR > 2011.

Covid-19: TITLE-ABS-KEY( "covid-19" OR "covid19" OR "coronavirus disease 2019" OR "2019-nCov" OR "Novel Coronavirus" OR "Novel Corona virus" OR "SARS-Cov-2") AND PUBYEAR $>2018$.

The search was last time updated on 24 May 2020 where it returned 5,907 items on SARS, 1,752 items on MERS and 11,859 items on Covid-19. Figures 1, 2, 3 respectively show the distribution of the studies on SARS, MERS and Covid-19, across subject areas. 


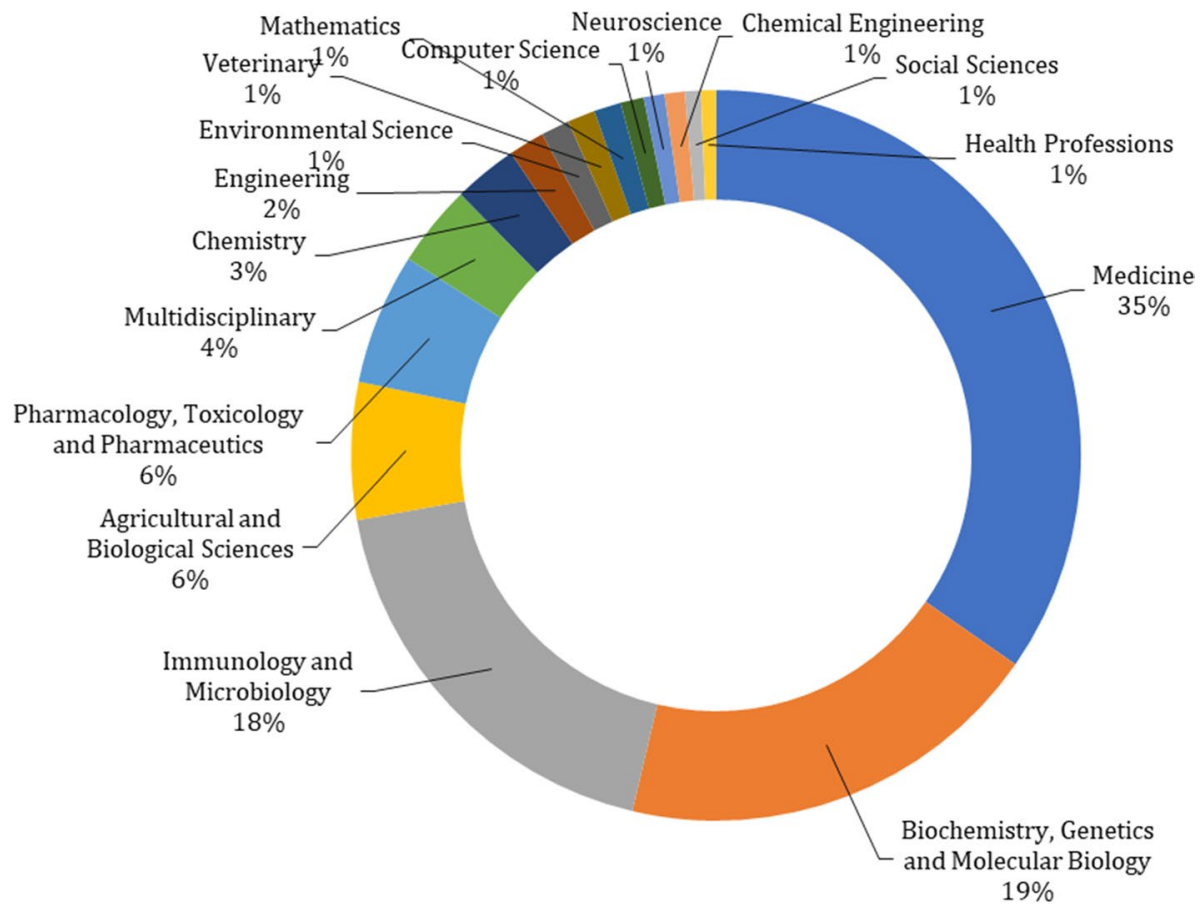

Fig. 1 Distribution of SARS studies across Subject Areas

Figure $4 \mathrm{a}$ also shows the composition of the Covid-19 literature in terms of the document types, demonstrating that only nearly $50 \%$ of the studies on this topic have so far been in the form of full-length articles, while letters, notes, reviews, and other document formats constitute a large portion (i.e. nearly half) of the literature on this topic at the time of this investigation.

Full records of the three datasets on SARS, MERS and Covid-19 were retrieved in CSV Excel format from Scopus, all on the same day. This included the citation information, bibliographic information, abstract and keywords, funding details and the references. The Scopus restriction of maximum 2000 document per export posed challenges for the retrieval of the SARS and Covid-19 datasets whose size were bigger than 2000 documents. For the SARS dataset, the challenge was circumvented by further limiting the search to specific years, in separate bundles, in a way that the size of each bundle was less than 2000 items, therefore allowing us to export the items of each bundle separately. The extraction of the Covid-19 dataset, however, posed a further layer of complication. Given that nearly all studies of Covid-19 have been published in one year, i.e. 2020, the year of publication could no longer be used as a criterion to form a set of mutually exclusive exportable bundles. To decompose the search outcome to non-overlapping bundles of 2000 documents or less, the following strategy was devised. The Document Type was used to initially limit the search to mutually exclusive (non-overlapping) categories. First, the search was limited to "Review or Short Survey or Erratum or Conference Paper or Data paper". This formed a set of 1267 documents which was extracted in one single export (see Fig. 4a for details of the number of items within each Document Type category). Subsequently, the search was 


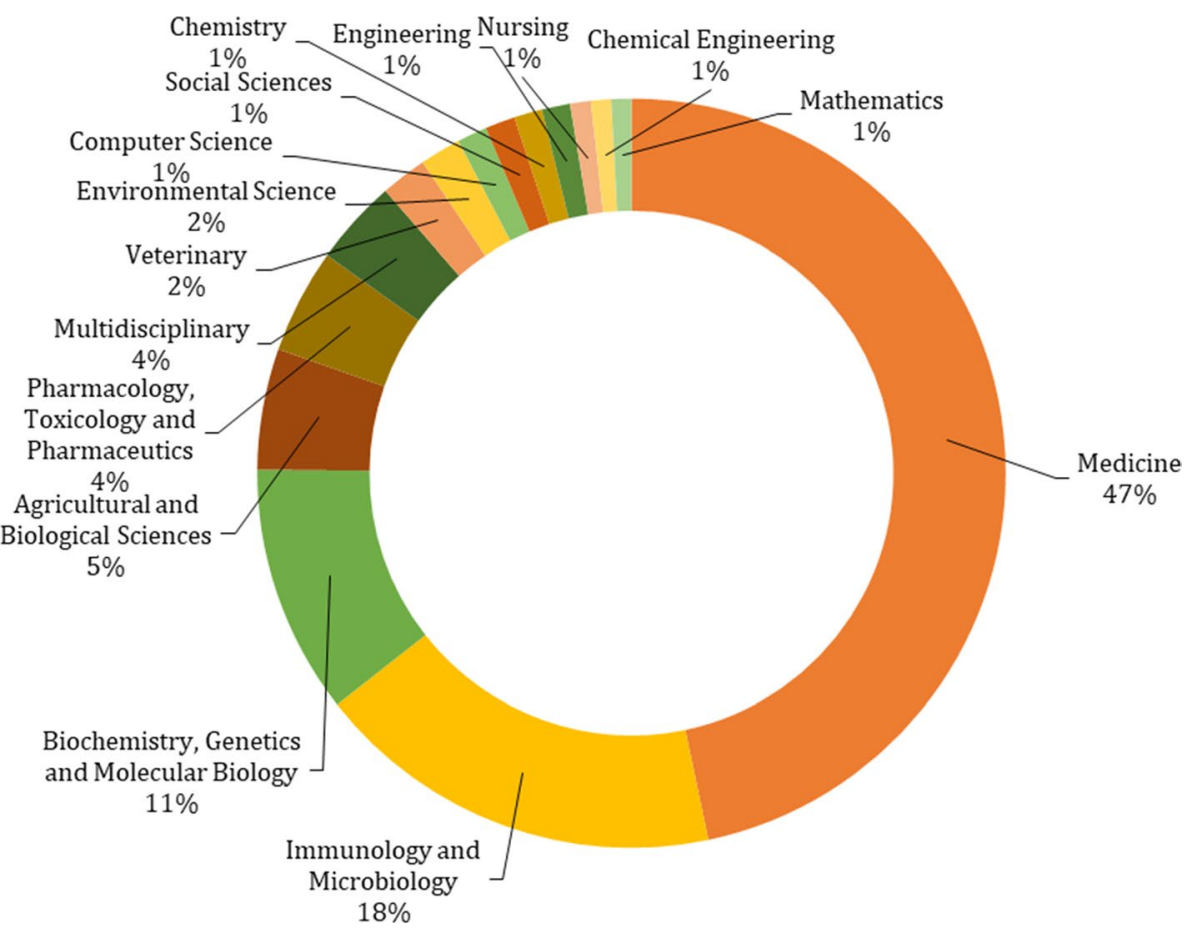

Fig. 2 Distribution of MERS studies across Subject Areas

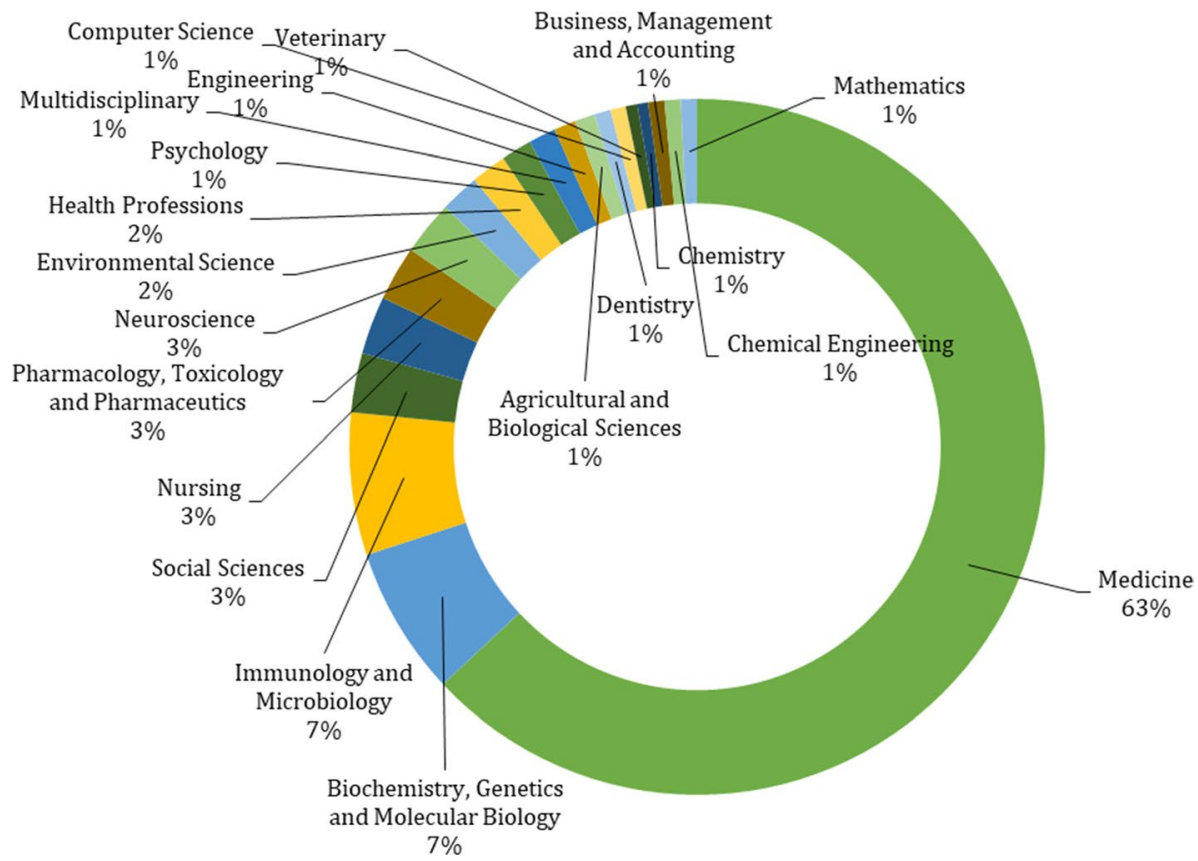

Fig. 3 Distribution of Covid-19 studies across Subject Areas 
(a)

Erratum, $70 \_$Conference Paper, 45

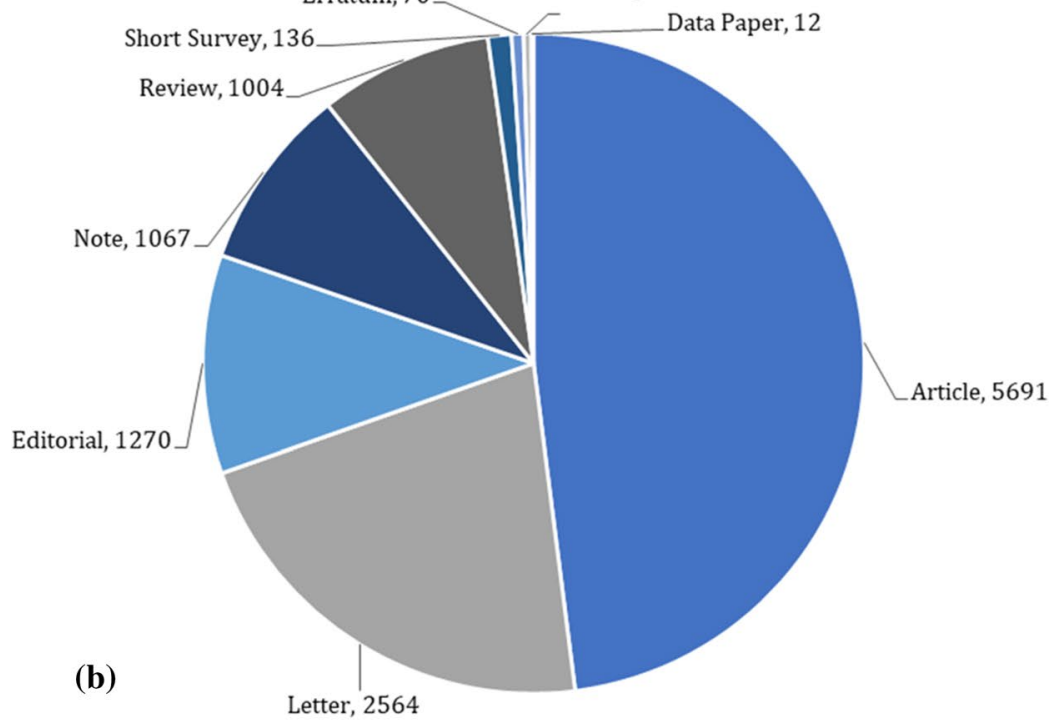

9000

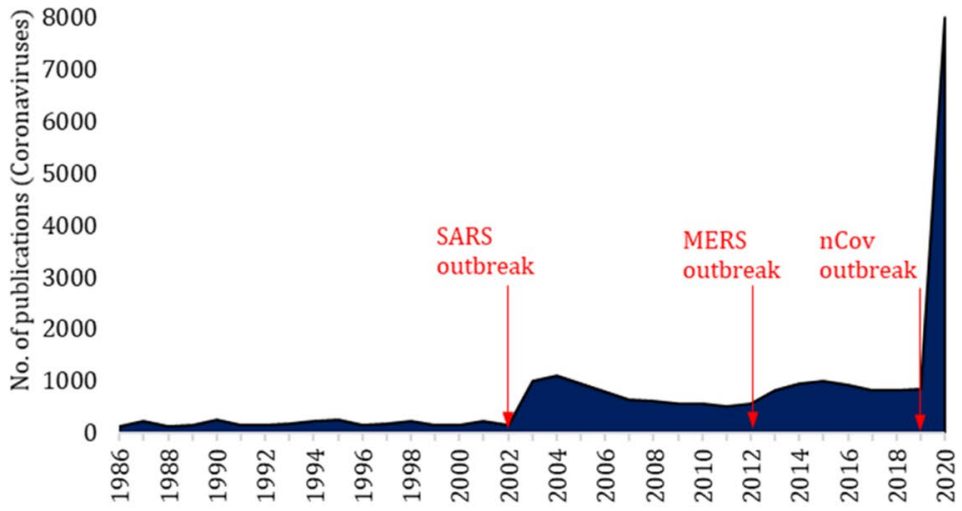

(c)

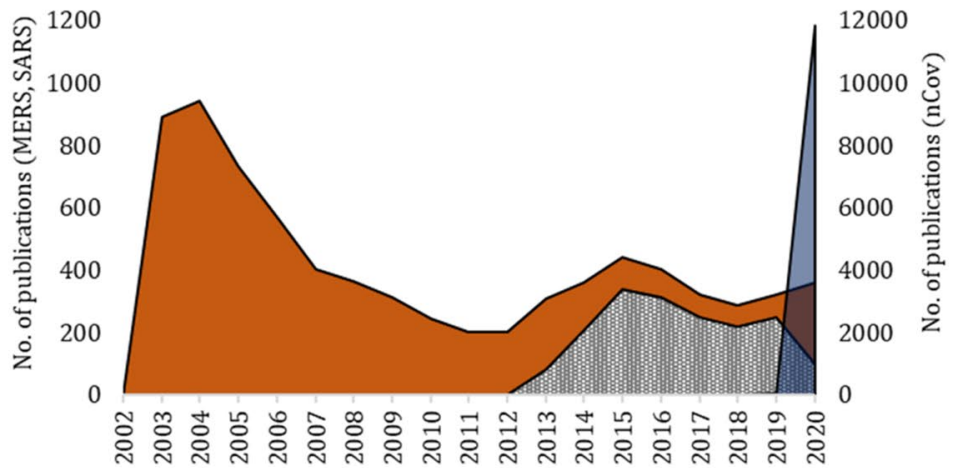

GMERS 口SARS 口nCov

Fig. 4 a Distribution of Covid-19 studies across Document Types, $\mathbf{b}$ Temporal distribution of the number of coronavirus studies, $\mathbf{c}$ Temporal distribution of the SARS, MERS and Covid-19 studies 
set back to the original and was limited to Notes (1067 items) and then to Editorial (1270 items). Each of these two subsets, being smaller than 2000 items, were exported separately. There were also 2564 documents of Letter type. This set was further decomposed to two mutually exclusive subsets based on the Publication Stage criterion (1539 Article in Press, and 1025 Final) and was retrieved in two separate exports. For the remaining 5691 Article documents, the following strategy was adopted. Of the 5691 items, 2944 were Article in Press and 2747 were Final. First, the 2944 Article in Press items were considered. The list of those studies was sorted as First Author (A-Z) and the first 2000 items were extracted in one export. Then the list was sorted as First Author (Z-A), i.e. the order was reversed, and the first 944 items were exported. A similar strategy was utilised to extract the remaining 2747 Final documents.

A supplementary search was also conducted on the general topic of coronaviruses using the string TITLE-ABS-KEY ( "coronavirus*") AND PUBYEAR $>1985$ which returned 24,620 documents on the same day. Only the data related to the number of documents by year was extracted for this search.

Figure $4 \mathrm{~b}$ shows the temporal distribution of the studies on the general topic of coronaviruses. The graph clearly shows spikes of publication coinciding with the years when SARS, MERS and Covid-19 outbreaks took place. The first spike is related to the SARS outbreak in 2002 which is reflected in an immediate and substantial increase in the number of publications on coronaviruses from 2002 to 2003. The increase continued, though at a slower pace, to 2004 and was then followed by a gradual decline till 2012. The 2012 MERS outbreak triggered another spike in the number of publications on coronaviruses, though not as large as that of SARS. The intensification of attention to this topic, this time, lasted for about three years till 2015 before another decline began. The spike of coronavirus studies prompted by the Covid-19 outbreak, however, seem to have been occurring at a completely different scale which can be deemed unprecedented in the history of coronavirus studies, and perhaps arguably in the history of science. The number of studies emerged in the first five months of 2020 nears an equivalent of the $70 \%$ of the total size of the coronavirus literature accumulated during more than 50 years (1968-2019). In Fig. 4c, the temporal distribution of the SARS, MERS and Covid-19 studies have been shown separately according to the three datasets explained earlier. Note that, the quantities associated with SARS and MERS are represented by the left vertical axis whereas that of Covid-19 is represented by the right vertical axis, while its scale is ten times bigger than the scale of the left axis.

The history of previous coronavirus research has suggested that the number of studies will likely keep rising for at least a few years before it peaks. But given the unprecedented magnitude of research and the explosive rate of publications since the begging of 2020, it would be interesting to observe whether this pattern would repeat itself and whether the peak would occur at an earlier or later stage compared to those of the previous outbreaks, a question whose answer will only be determined by time.

\section{Keyword co-occurrence analyses}

The co-occurrence of keywords associated with the SARS, MERS and Covid-19 literatures were analysed using VOSViewer (Van Eck and Waltman 2010). Each analysis was performed on the separate set of data associated with the literature of interest. The maps of keyword co-occurrences associated with SARS, MERS and Covid-19 literatures are 


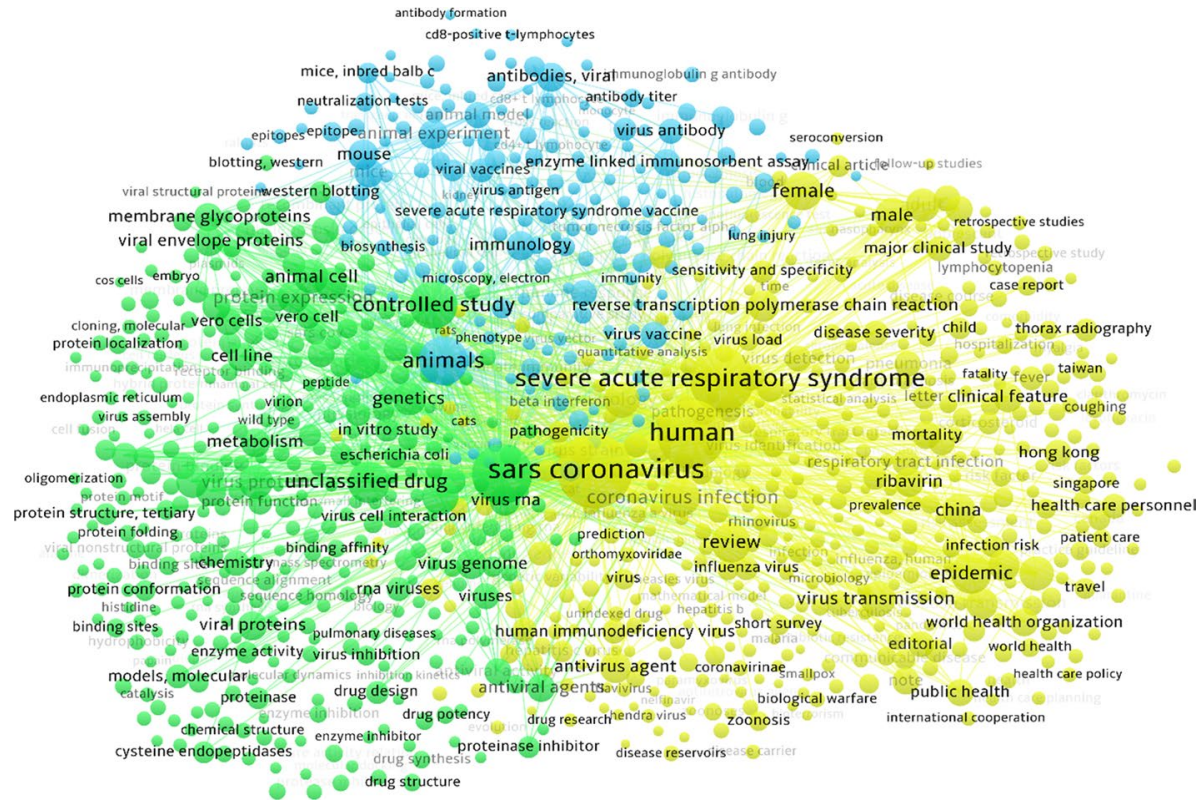

Fig. 5 The map of keyword co-occurrences associated with the SARS literature

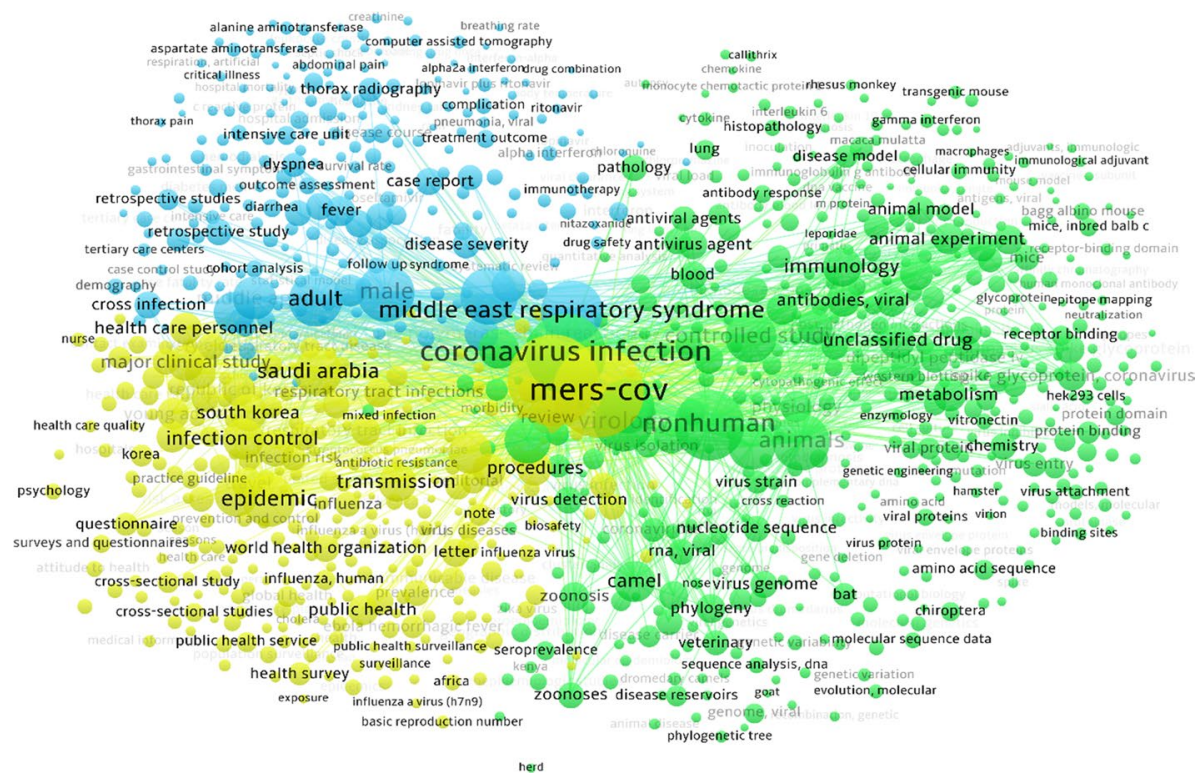

Fig. 6 The map of keyword co-occurrences associated with the MERS literature

provided in Figs. 5, 6, 7 respectively. The minimum number of occurrences for the keywords to be included in the map was set to 5 in all three cases. The number was chosen on the basis of the clarity of the resultant clusters. The unit of analysis has also been set 


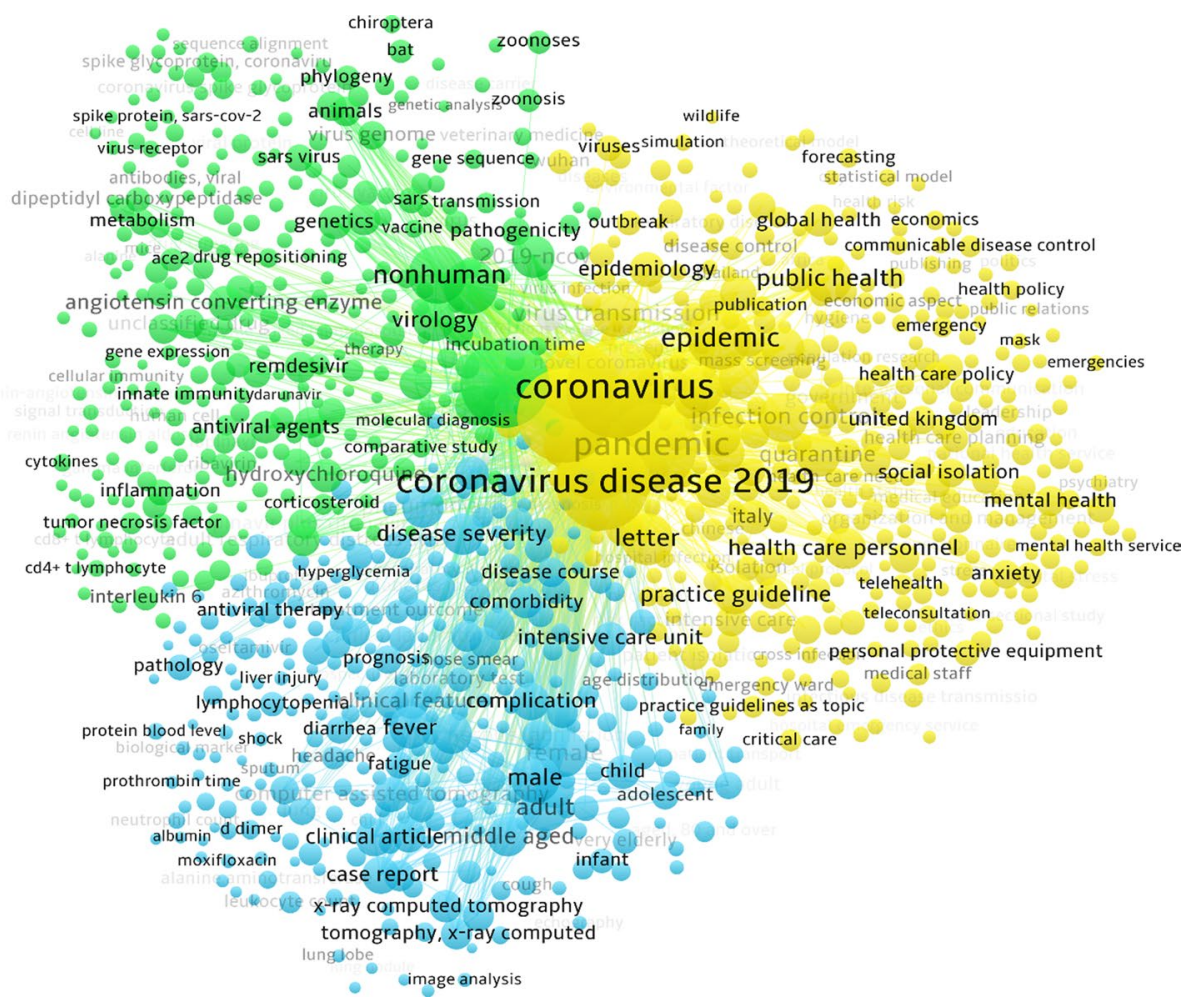

Fig. 7 The map of keyword co-occurrences associated with the Covid-19 literature

to all keywords (that includes both author and index keywords) and the method of counting was binary counting. Figs. 17, 18 in the "Appendix" illustrate the map associated with the SARS literature overlaid respectively with the average year of publication and average number of citations associated with the studies in which these keywords have occurred. Figs 19, 20 present the counterpart outputs for the MERS literature analysis. Fig. 21 is a heatmap of Covid-19 keyword co-occurrence and Fig. 22 overlays the Covid-19 map of keywords in Fig. 7 with the colour-coding of the average number of citations. Given that almost all studies of Covid-19 are 2020 items, the colour-coding related to the average publication year was forgone with respect to this literature. Maps of term occurrences based on the analysis of the title and abstract of studies on SARS, MERS and Covid-19 have also been presented in the "Appendix" in Figs. 23, 24, 25 respectively. While the below analysis focuses mainly on the interpretation of the keyword maps, similar patterns are by-and-large observable through analysis of the title and abstract terms of these studies.

With respect to each of the three literatures, three distinct clusters of keywords were identifiable. These clusters showed certain patterns of commonality across the three datasets. Each map presents a distinct cluster of keywords that seem to be associable to the studies related to public health emergency management and the prevention of epidemic. This cluster has been assigned a yellow colour in all three maps in Figs. 5, 6, 7 Here, this is referred to as Cluster (i). In this cluster, one can observe terms such as those associated with general public health including "wold health organisation", "public health", "public 
health service", "global health", as well as those associated with disease outbreaks including "emergency", "health risk" "epidemics", "pandemic", "outbreak", "viral diseases", "virus infection", "communicable disease", "transmission", "travel". Terms representing measures of emergency severity also appear in this cluster including "mortality", "fatality", "morbidity", "infection risk". This cluster also includes terms that are linked to the prediction of disease propagation. These are terms such as "mathematical model", "modelling", "simulation", "statistical model" and "prediction" that have commonly occurred in this cluster. The cluster includes terms affiliated with measures of disease control and spread prevention such as "(social/patient) isolation", "quarantine", "hygiene", "handwashing", "prevention", "infection control", "(population) surveillance", "mass screening", "(face) mask", "contact tracing". The cluster also represents keywords that are attributable to public policy making and social protection such as "health care planning", "health care policy", "health care quality", "leadership", "disaster planning" and "polices".

The Cluster (i) of keywords also have distinctly and commonly (across all three datasets) represented keywords that are attributable to the studies on mental health impacts of the epidemic. These are keywords such as "mental health (service)", "psychiatry", "psychology", "mental stress", "anxiety", "fear", "mental disease". These studies have often used methods such as "questionnaire(s)" and "survey(s)" that have commonly reflected in this cluster across the three literatures. Issues surrounding the safety of medical facilities and medical staff also appear to have been addressed mainly by studies whose keywords are attributable to this cluster. These studies have generated keywords such as "health care personnel", "nurse(s)", "medical staff', "hospital", "health care facility", "personal protective equipment" that are distinctively observable in Cluster (i) of keywords across all three datasets.

The economic aspects of the epidemics also seem to have been addressed particularly by Covid-19 as reflected in Cluster (i) of the Covid-19 literature. These have been reflected in terms such as "economics", "economic aspect", which have occurred frequently enough in Covid-19 studies to appear distinctly on the map. The presence of such cohort of studies is, however, not as clearly identifiable on the SARS and MERS maps as is it on the Covid19 map. This could be explained by the greater magnitude of the societal impact of Covid19 outbreak compared to SARS and MERS.

The names of the countries and regions have almost invariably appeared in Cluster (i) across all three datasets. In certain cases, the country names that have occurred most often are those from which the outbreaks originated or those that suffered most from the impact of the outbreak. For example, "Saudi Arabia" appears quite distinctly on the Cluster (i) of the MERS dataset. Similarly, the presence of the names of south-east Asian countries/regions such as "China", "Hong Kong", "Taiwan", "Singapore" on the Cluster (i) of the SARS map, or the term "Wuhan" on the Cluster (i) of the Covid-19 map are quite pronounced. The occurrence of the names of countries also could be a reflection of the early (case) studies that have addressed the local impacts/spread of the outbreaks within their own society. On the issue of early studies, the terms "letters", "editorial", and "review" (which have intentionally been kept on the maps) seem to also have distinctly occurred in Cluster (i) of each literature which is an indication that this cluster includes early studies that appeared at a time where the amount of data and clinical trials were insufficient for full-length articles. An inspection of the Figs. 18, 20 does, in fact, confirm this hypothesis at least in association with the SARS and MERS literature, that the Cluster (i) of keywords represent studies that on average emerged earlier during the developments of their respective literatures. Figures 19, 21, 23 that have illustrated the colour-coding of the average number of citations on the maps also show that, although Cluster (i) is associated with the early studies that generally preceded studies of the two other clusters 
and although it represents the largest variety of topics compared to the two other clusters, it is also associated with the studies that, on average, been the recipient of a lesser number of citations when compared to the two other clusters. This pattern appears to have commonly occurred across all three datasets.

A second cluster of keywords associated with each of the three literatures were also discovered that is attributable to the studies on the chemistry and physiology of the virus, or viral pathogenesis, or in other words, the chemical constitution of the virus (Knight 1954), a sector of virology that investigates the biological processes and activities of viruses that take place in infected host cells and result in the replication of a virus. This cluster has been assigned the green colour in the maps of Figs. 5, 6, 7. According to the maps, the most distinct terms associated with this cohort of virology studies on SARS, MERS and Covid-19 are terms such as "virus protein", "virus entry", "chemistry", "metabolism”, "physiology”, "pathology", "cell line", "(virus/viral) protein(s)", "molecular model(s)", "virus genome", "virus rna", "virus replication", "mutation", and "enzyme activity". As this sector of studies often use "animal model(s)", terms such as "animal cell", "animal experiment", "controlled study", "mice" and "mouse" have frequently appeared in the representative Cluster (ii). In reflection of the fact that these cohort of studies ultimately seek "drug design", in addition to generic common terms such as "drug design/potency/structure/synthesis", the names of the specific potential drugs that have been investigated in relation to each disease have appeared in this cluster. This includes terms such as "hydroxychloroquine" or "remdesivir" on the Covid-19 map. An inspection of the maps overlaid with the average year of publications for SARS and MERS in Figs. 18, 20 in the "Appendix" suggests that, on average, this cohort of studies are generally the last to appear in the published domain compared to the two other major clusters, but, according to Figs. 19, 21, 23, they receive relatively high citations on average.

A third and relatively smaller cluster of keywords was commonly identifiable in relation to each three literatures. This cluster has been visualised in blue across all three maps of keyword co-occurrence. The studies represented by this cluster of keywords, here referred to as Cluster (iii), appear to have been more closely focused on the developments of antibodies and vaccines. The terms "treatment", "treatment outcome", "disease severity", "antiviral therapy", "prognosis", "drug safety", "prospective/retrospective study", "immunology", "immunotherapy", "innate immunity", "immune response", "virus/viral vaccine(s)", "virus/viral antibody" across studies of this cluster. Terms affiliated with studies related to treatments and clinical care of respiratory patients also appear in this cluster. This includes terms such as "artificial ventilation", "intensive care unit", as well as symptom and organ terminologies associated with each disease, terms such as "fever", "headache", "diarrhea", "lung (injury)", "coughing", "liver injury", "kidney". Terms affiliated with cohort analysis studies have appeared in this cluster of the maps associated with each literature. This is reflected in terms such as "female", "male", "child", "infant", "young adult", "adult", "age", "middle aged", "pregnant", "pregnancy".

\section{Bibliographic coupling and citations of journals}

Bibliographic coupling of the studies on SARS, MERS and Covid-19 were analysed at the level of their sources/journals. Figures 8, 9, 10 show the maps of journal bibliographic coupling associated with SARS, MERS and Covid-19 literatures respectively. The node sizes are proportional to the number of documents published by the corresponding sources and the thickness of the links are proportional to the degree of 


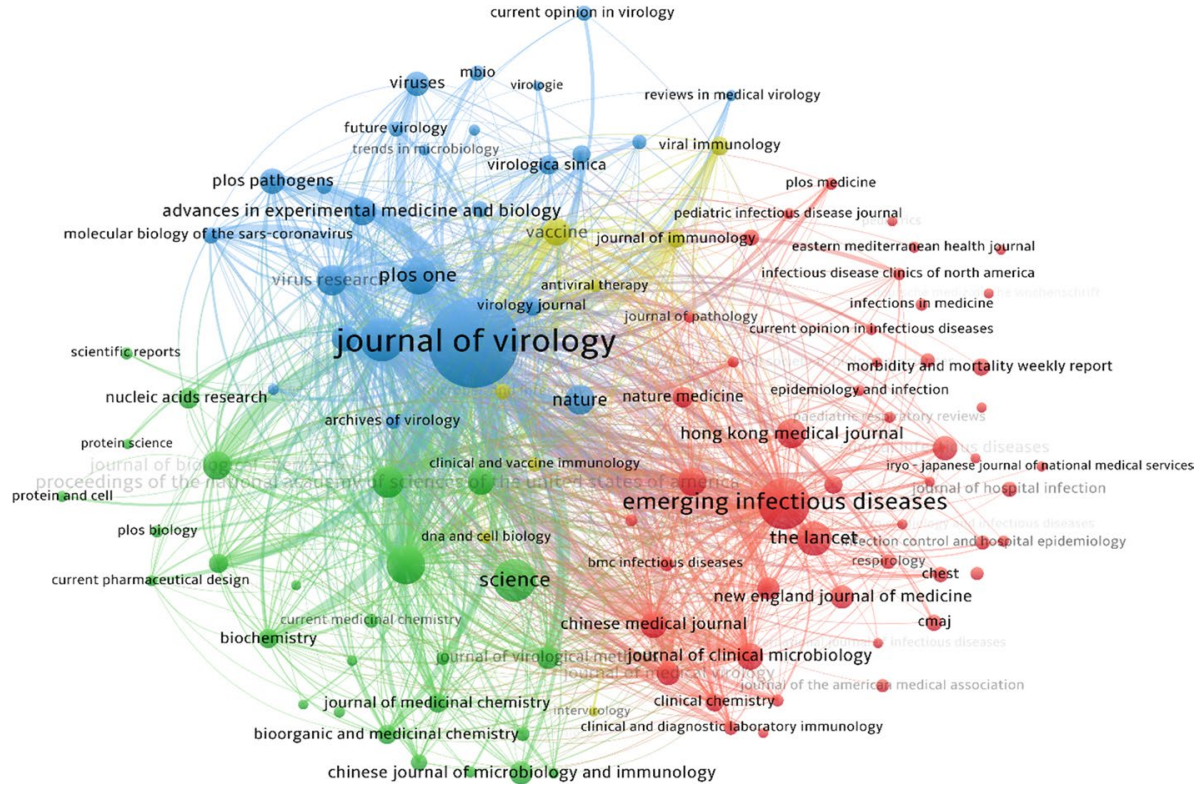

Fig. 8 The map of bibliographic coupling of sources associated with the SARS literature

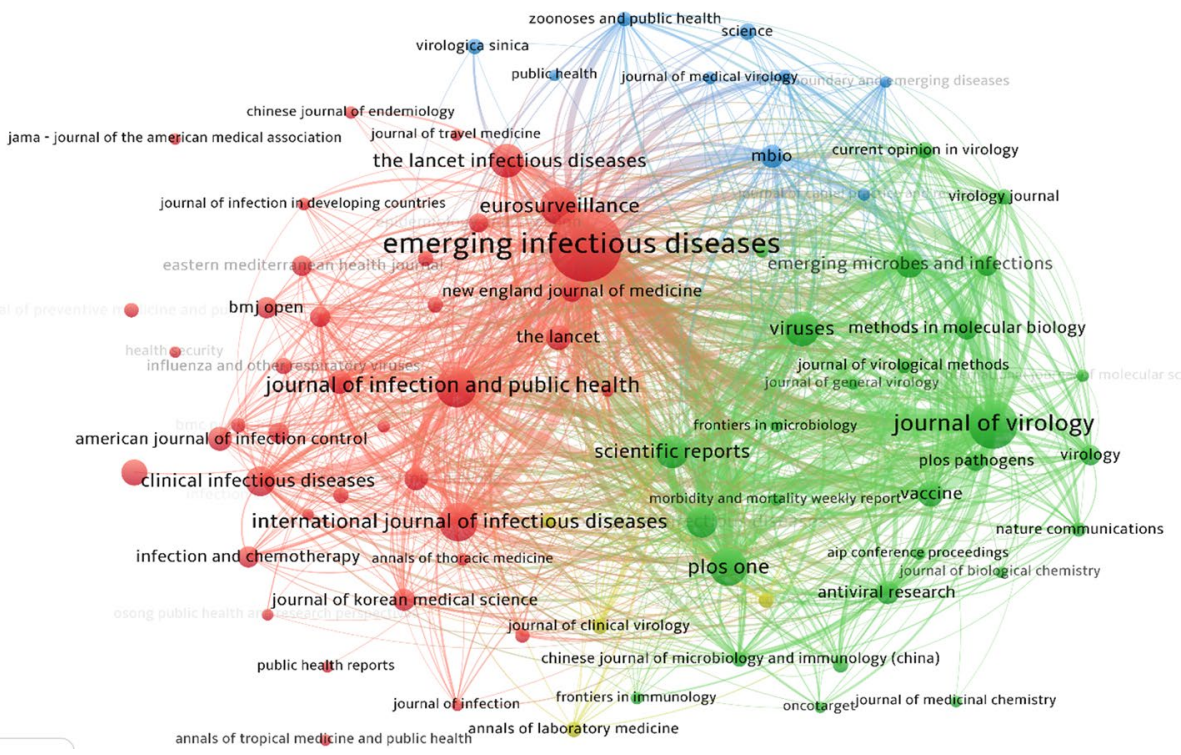

Fig. 9 The map of bibliographic coupling of sources associated with the MERS literature

bibliographic couplings between the pairs of sources connected by each link. The minimum number of documents associated with each node/journal to appear on the map has been set to 10 . The number has been set on the basis of maximising clarity of the 


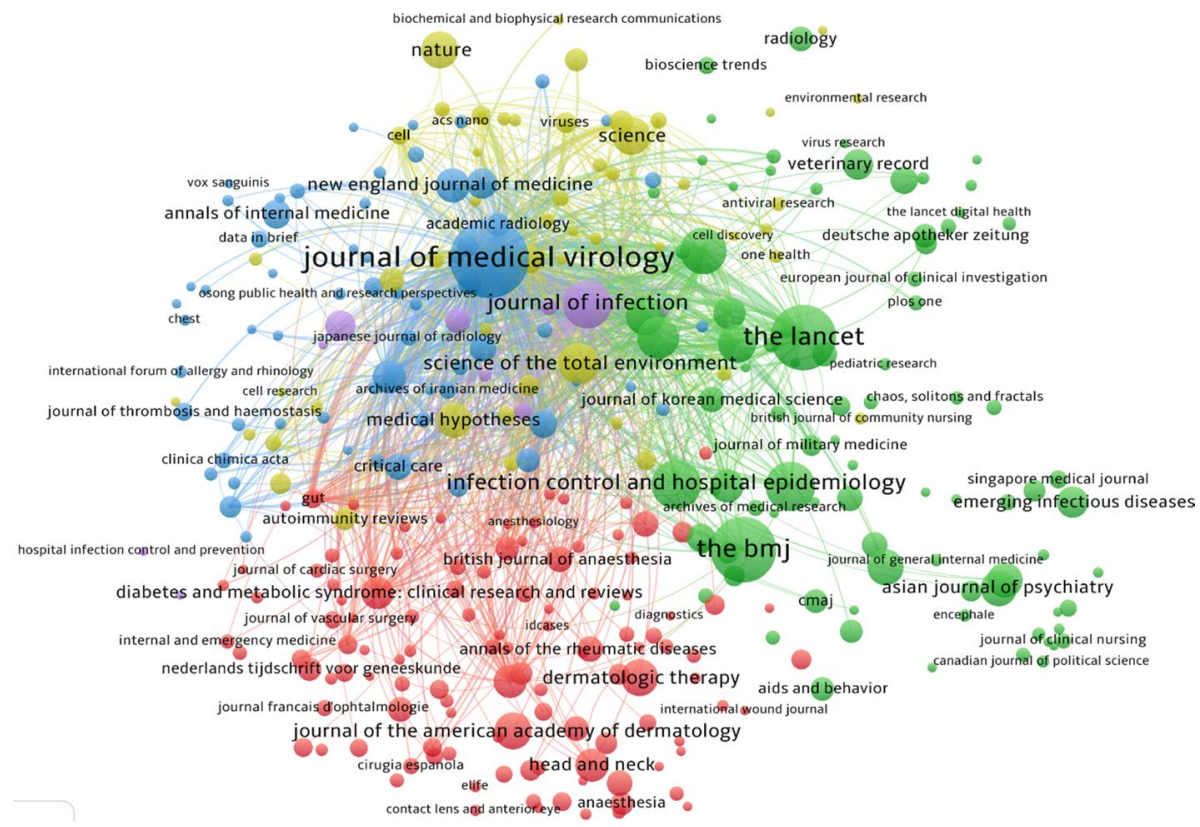

Fig. 10 The map of bibliographic coupling of sources associated with the Covid-19 literature

resultant clusters on the maps. No minimum strength was set for links to be visualised on the map.

A first-glance comparison shows that while the maps associated with SARS and MERS are well connected, connections across the Covid-19 map are rather sparse. Both the SARS and MERS maps include three distinct and well-defined clusters of bibliographically coupled journals in addition to one minor and smaller cluster. These clusters show relatively strong degrees of inter-connectivity and coherence, whereas, this feature is not shared by the Covid-19 map. The observation is understandable considering the fact that the SARS and MERS literatures are relatively well established and have stabilised given that they have both been under development over a period of several years, whereas the Covid-19 literature is an emerging and evolving field. Newly published Covid-19 studies do not seem to be sharing many references as of yet, which explains why their coupling connections are relatively weak. The comparison also suggests that the Covid-19 studies are generally scattered across a broader variety of journals and subject areas, as opposed to the SARS and MERS publications that seem to have been concentrated across a smaller set of specialty (mostly medical) journals. This is also consistent with our observations from Figs. 1, 2, 3 showing explicitly that studies of Covid-19 are scattered across a broader variety of subject areas compared to the SARS and MERS literatures. Though not shown in Fig. 3, due to the respective values being smaller than $1 \%$, journals in the following subject areas (those that are deemed minor areas in relation to Covid-19 literature) have each published a relatively considerable number of studies on this topic (a feature that is not necessarily shared 
by the rest of the literature on coronaviruses): Arts and Humanities (110 items, ${ }^{2}$ where the most active journal has been Social Anthropology (24 items) covering topics such as "climate change reactions" (Bychkova 2020), or "legal voids linked to declared states of emergency" (Karaseva 2020)), Economics, Econometrics and Finance (84 items, with Economic and Political Weekly (36 items) being the most active journal of that category, covering topics such as "food supply chains" (Reardon et al. 2020), "economic stimulus packages" (Mulchandani 2020) or "reverse migration" (Dandekar and Ghai 2020)), Physics and Astronomy (77 items, where Chaos Solitons and Fractals (16 items) has been the most active publication outlet, covering topics such as "mathematical models for forecasting the outbreak" (Barmparis and Tsironis 2020; Bekiros and Kouloumpou 2020; Boccaletti et al. 2020; Ndaïrou et al. 2020; Postnikov 2020; Ribeiro et al. 2020; Zhang et al. 2020)), Energy (67 items, with International Journal of Advanced Science And Technology (44 items) being the most active journal in that category, covering topics such as "Flexible work arrangement in manufacturing" (Sedaju et al. 2020)), Material Sciences (57 items, with ACS Nano (10 items) being the most active outlet in that category, covering topics such as "3-D printed protective equipment" (Wesemann et al. 2020)), Decision Sciences (23 items, with Lancet Digital Health (8 items) and Transportation Research Interdisciplinary Perspectives (4 items) being the most active outlets in that category, covering topics such as "the effect of social distancing on travel behaviour" (De Vos 2020) or "the implementation of drive-through and walk-through diagnostic testing" (Lee and Lee 2020)), Earth and Planetary Sciences (22 items, with Indonesian Journal of Science and Technology (8 items) being most active in that domain, covering topics such as "the deployment of drones in sending drugs and patient blood samples" (Anggraeni et al. 2020)).

A prominent source that seem to have consistently published a substantial portion of studies on SARS and MERS is Journal of Virology. This journal, however, has not published a considerable number of studies on Covid-19, and with only 8 publications on this topic at the time of writing this article, it does not have a strong representation on the map of Covid-19. The Lancet and Science, however, are two major outlets notably identifiable on all three maps. For Covid-19 studies in particular, journals such as Journal of Medical Virology, The BMJ, The Lancet, Journal of Infection, Science, Nature, Science of the Total Environment and Medical Hypotheses have been most notable outlets of publications so far. Some of these outlasts, such as Science of the Total Environment and Medical Hypotheses, do not have a strong representation on the maps associated with the SARS or MERS.

In terms of the bibliographic coupling of the sources for SARS publications, the strong relation between Journal of Virology and Virology, and to lesser extent, with Emerging Infectious Diseases are outstanding. For MERS publications, the strong bibliographic coupling of publications between Emerging Infectious Diseases and Journal of Virology is most outstanding. For Covid-19 publications, the one outstanding bibliographic coupling relation is one that exits between Journal of Medical Virology and Journal of Infection.

The analyses of journal citations also showed similar patterns of scatter and relatively cohesive clusters in relation to the Covid-19 literature compared to the welldefined clusters of journal citations for SARS and MERS literatures. Consistent with the previous observation with respect to journal bibliographic coupling, the Covid-19 literature seems to be also much less cohesive in terms of its journal citation networks, when compared to the SARS and MERS literatures. As discussed earlier in relation to bibliographic couplings, this could also be partly explained by the fact that Covid-19

\footnotetext{
${ }^{2}$ Note that these Figures are based on a renewed Scopus search on 30 May 2020 when the total number of Covid-19 studies was sitting at 13,700.
} 


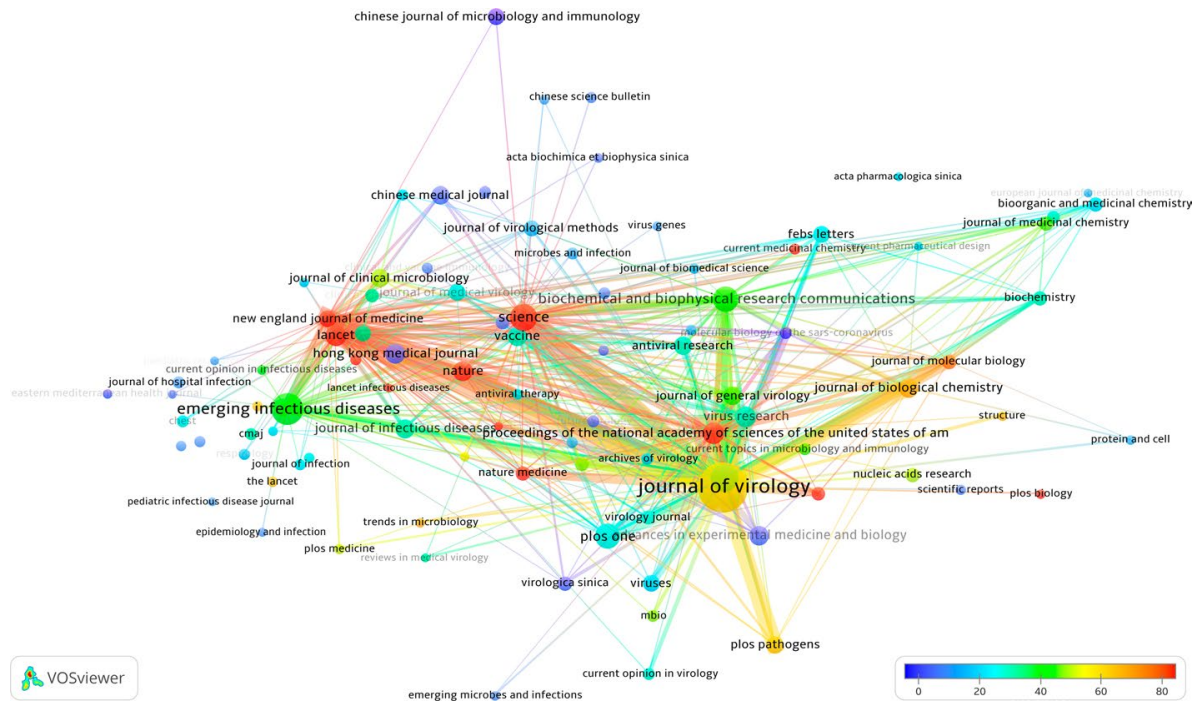

Fig. 11 The map of strongest citation network of sources for SARS overlaid with the colour-coding of the average citation per document

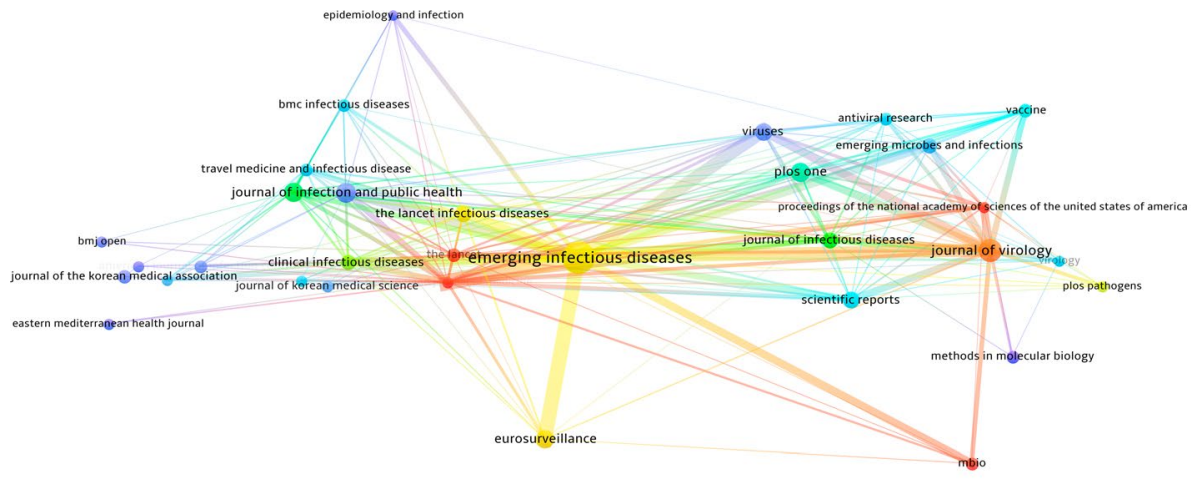

禹 vosviewer

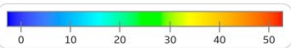

Fig. 12 The map of strongest citation network of sources for MERS overlaid with the colour-coding of the average citation per document

papers are scattered across a more diverse range of journals and broader variety of subject categories. For the maps of journal citation relations presented in Figs. 11, 12, 13, only the strongest citation relations have been visualised by increasing the minimum strength threshold for the citation links between pairs of journals to show on the map. The nodes have also been overlaid with the average citation colour coding. For SARS, the maps present very strong citation relations between publications in Journal of Virology and those of Virology, PNAS and Science (and to a lesser extent, with Emerging Infectious Diseases, The Lancet and New England Journal of Medicine). For MERS 


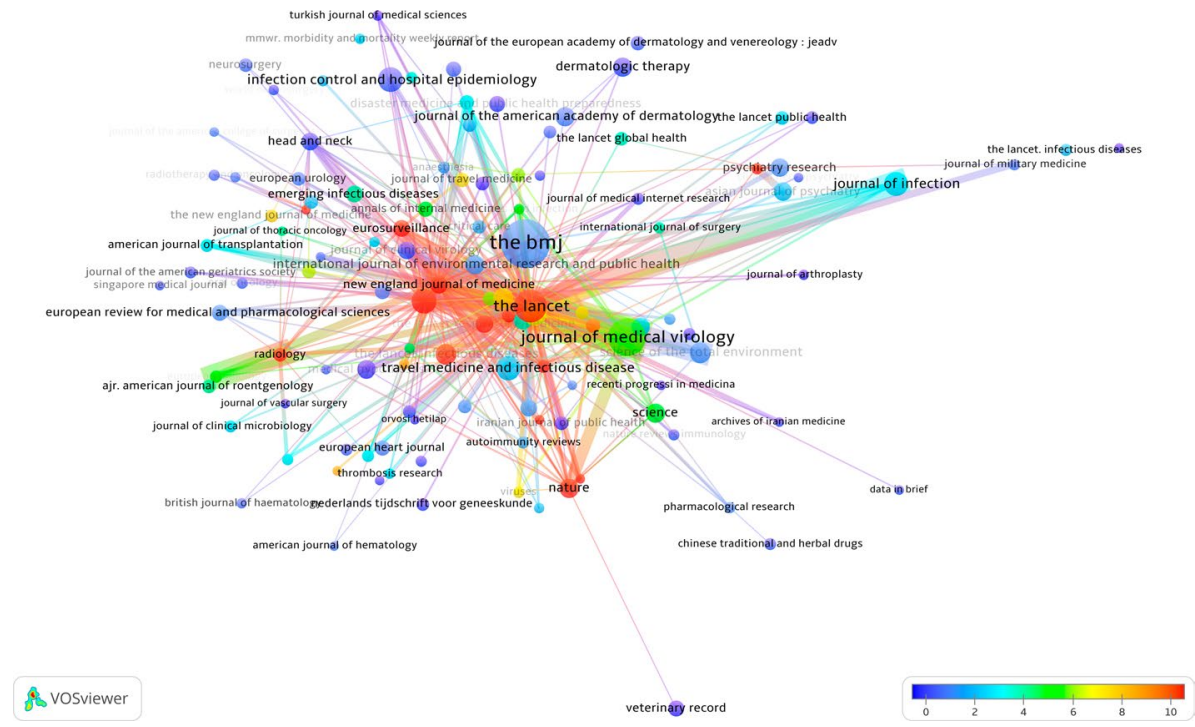

Fig. 13 The map of strongest citation network of sources for Covid-19 overlaid with the colour-coding of the average citation per document

publications, the strong citation relation between Emerging infectious Diseases and New England Journal of Medicine are the ones that most stand out. Also, for the Covid-19 literature, three pairs of strong citation relations are identifiable: first one between Journal of Medical Virology and The Lancet, second one between Journal of Medical Virology and New England Journal of Medicine, and third one between European Radiology and Radiology.

In Figs. 27, 31 in the "Appendix", the nodes of the bibliographic coupling maps have been colour-coded by the average year of publications and the average citations per document associated with the journals that each node represent (except for the Covid-19 map that has only been overlaid with the average citations). According to these maps, Emerging Infectious Diseases and The Lancet have been a major source of publications for early studies on both SARS and MERS. This pattern for The Lancet seems to have extended to Covid-19 studies as well, as this journal has published a substantial portion of the early studies on this topic. For SARS, the strong representation of Chinese Medical Journal and Chinese Journal of Microbiology and Immunology among the journals that published early studies are notable, a pattern that could be explained by the geographical origin of the SARS outbreak. Such pattern is to a less obvious extent also observable with respect to the MERS literature. It reflects on the MERS map through representations of journals such as Eastern Mediterranean Health Journal and Saudi Medical Journal by colours associated with early publications.

In terms of the average number of citations, studies published by The Lancet have consistently and across all three literatures received high citations. For the SARS literature in particular, other sources whose publications on this topic received high numbers of citations are New England Journal of Medicine, PNAS, Current Medical Chemistry, Journal of Pathology, Plos Biology, and Nature Reviews Microbiology. The counterpart outlets associated with the MERS literature are Journal of Virology, Science, mbio, 


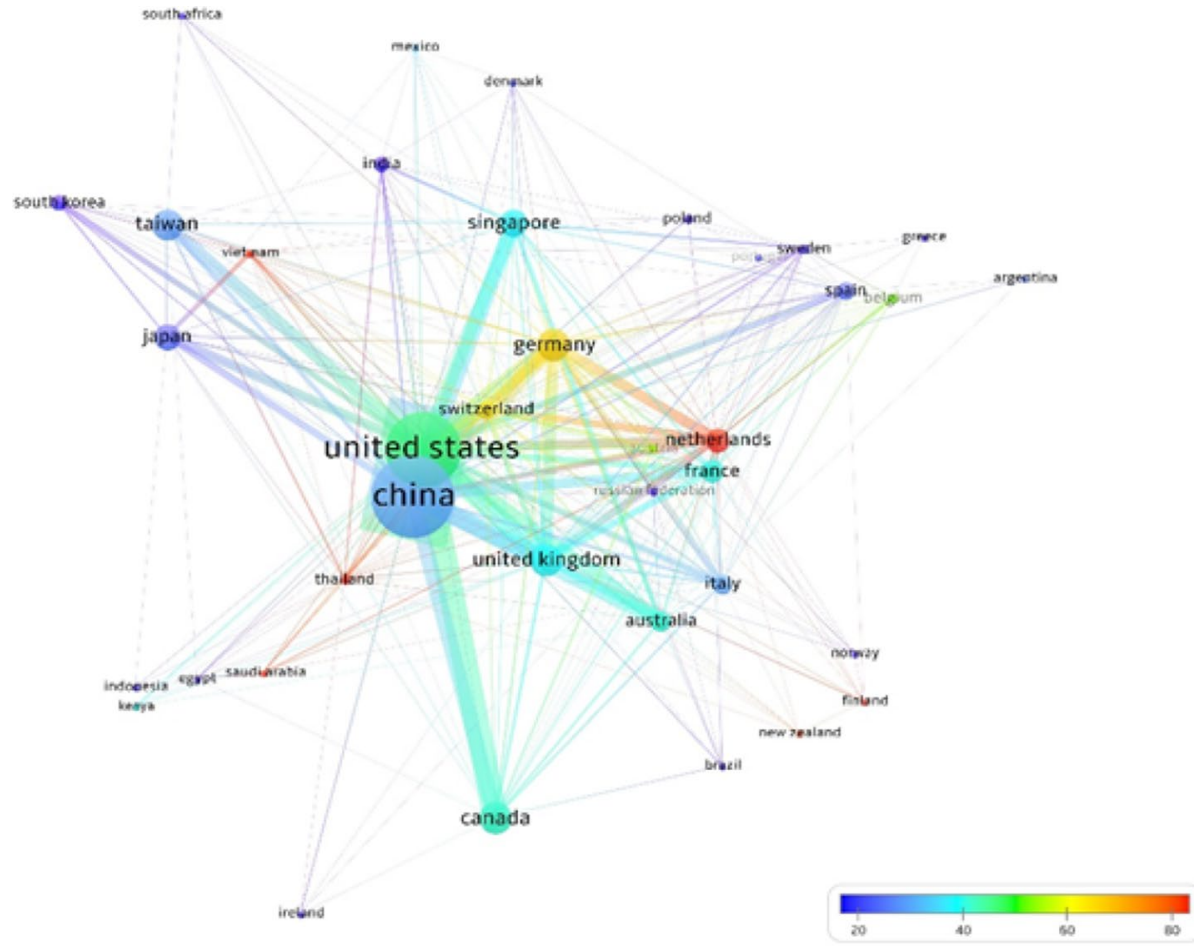

Fig. 14 The map of country co-authorships associated with the SARS literature

and New England Journal of Medicine. For Covid-19 studies, publications of Nature, Eurosurveillance, The Lancet Infectious Diseases, JAMA, The New England Journal of Medicine, and Radiology have on average been among the most cited studies.

\section{Co-authorship between countries}

Collaborations of authors aggregated at the level of the countries were also analysed with respect to the SARS, MERS and Covid-19 literatures. Outputs of the analysis are presented in Figs. 14, 15, 16, for SARS, MERS and Covid-19 respectively. In each map, the size of nodes, each corresponding with a country, are proportional to the number of published documents with an author affiliated with the institutes of those countries. The links connecting the nodes indicate co-authorships between authors residing in the countries, while the thickness of the links represent the strength (i.e. frequency) of the co-authorships. The colour assigned to each node represents the average number of citations that studies emerged from countries have received. The minimum number of documents for country names to appear on the maps has been set to 5, a number chosen based on the criterion of the quality of the resultant maps.

Comparison across the three maps of co-authorships shows a pattern of author involvement from the regions where each viral outbreak originated. Studies authored by researchers affiliated with Chinese institutes are well represented in all three cases, but clearly 


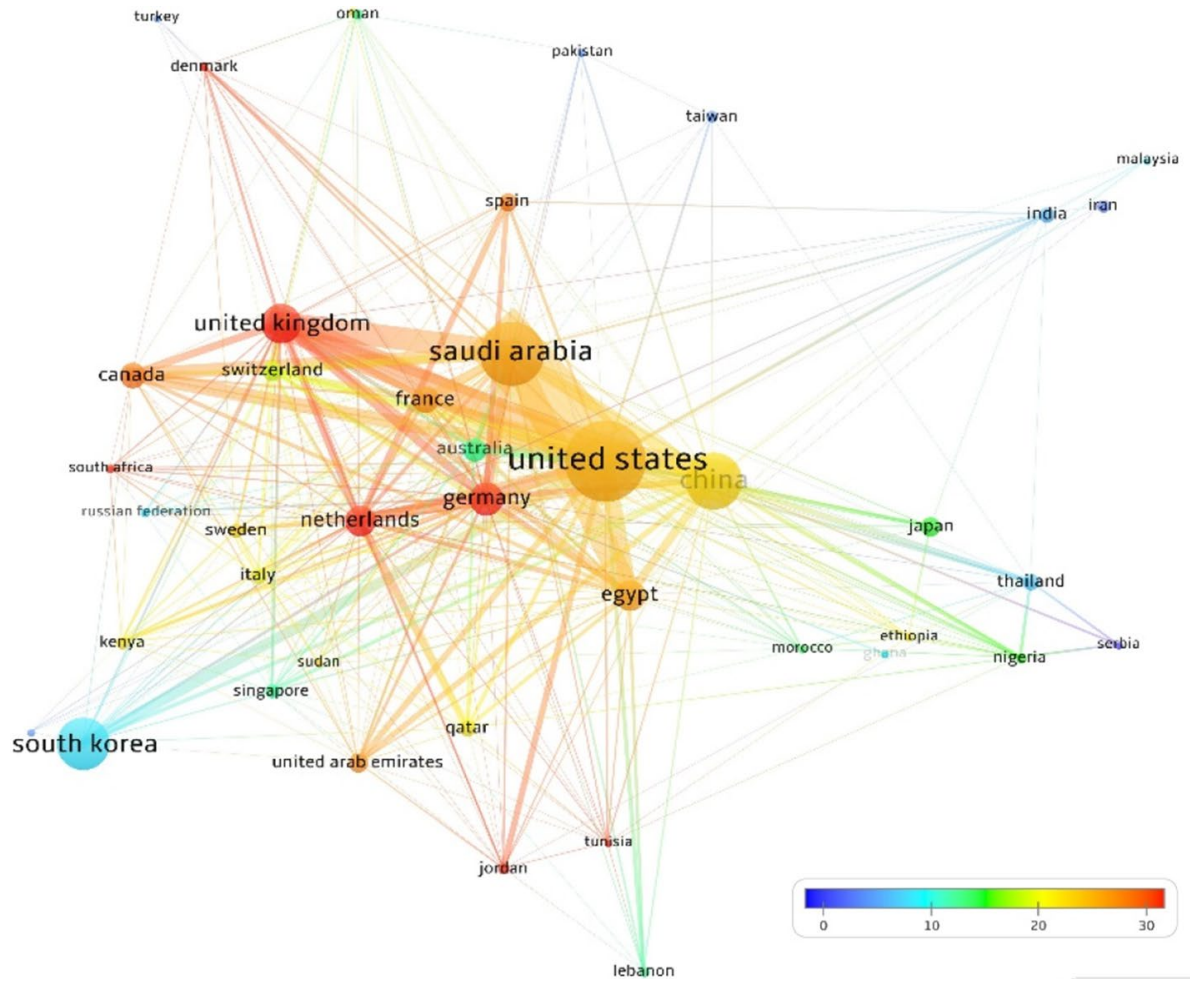

Fig. 15 The map of country co-authorships associated with the MERS literature

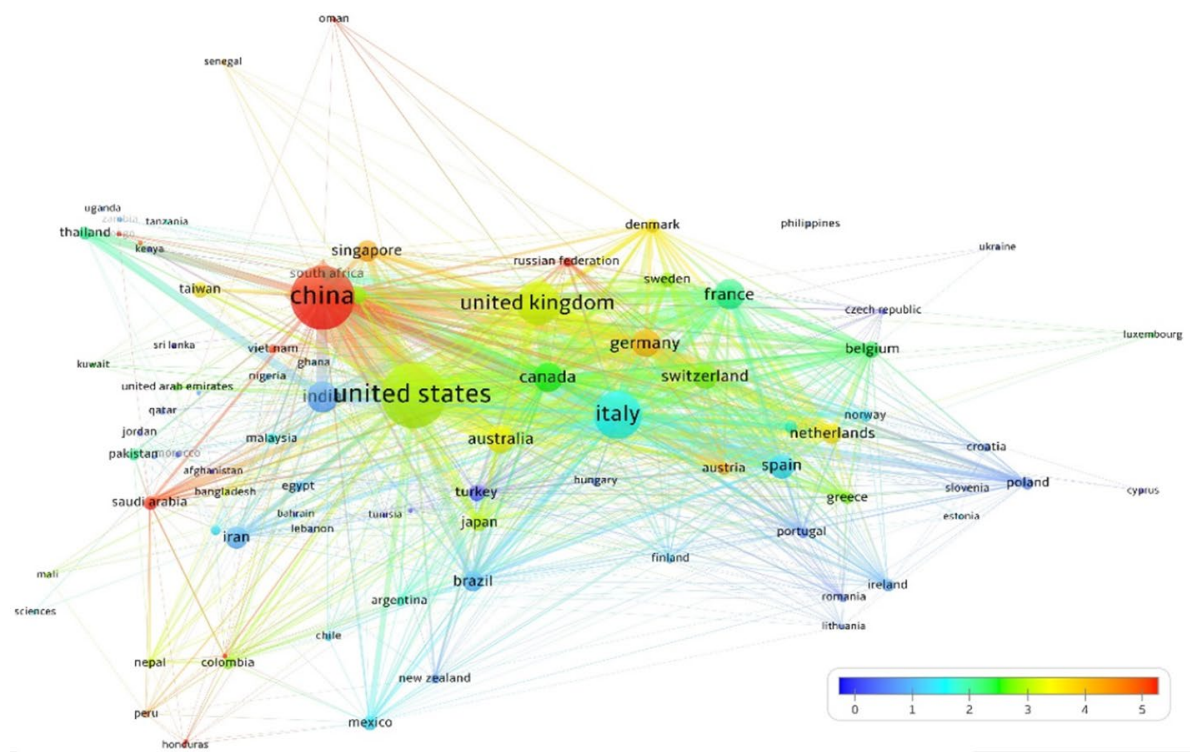

Fig. 16 The map of country co-authorships associated with the Covid-19 literature 
more so with respect to the SARS and Covid-19 literature, diseases whose first cases were recorded in China. The involvement of Chinese authors is relatively less pronounced with respect to the MERS studies whose origin was in the Middle East. Instead, with respect to the MERS literature, it appears that authors affiliated with institutes in Saudi Arabia have been exceptionally overrepresented in the publications. This is also, to a lesser extent, the case with Egypt being markedly presented on the MERS map of the country co-authorships.

On SARS studies, Chinese authors have most strongly collaborated with authors residing in the United States, followed by authors from Germany, Taiwan, Singapore, Japan, France, Australia, United Kingdom and Canada. The SARS network of collaborations for the authors affiliated with Institutes in the United States has been, by and large, similar to that of China. Except, South Korea, The Netherlands, Italy and Spain are also strongly represented in the collaborations with the United States.

The map of co-authorships associated with the MERS literature presents the names of many Middle Eastern countries including Saudi Arabia, Egypt, Lebanon, Iran, Tunisia, Qatar, Oman, Jordan, and United Arab Emirates which is a clear indication into the exceptionally strong representation of the authors from this region in these studies. The strongest network of co-authorships on this topic are observed between authors from the United States and those of Saudi Arabia, followed by China, United Kingdom, Egypt, South Korea, Canada and the Netherlands. The closest collaborators of Chinese authors on this topic, after the United states, have been from Saudi Arabia, United Kingdom and Egypt. The closest collaborators of Saudi Arabia, after the United States, have been Egypt, China and United Kingdom.

The Covid-19 map presents a considerably higher number of country names compared to maps of the SARS and MERS literatures. It clearly shows that authors from a larger number of countries have become involved in studies of Covid-19, compared to the research on SARS and MERS that have apparently engaged a lesser number of countries and institutes. China, on the topic of Covid-19, has a very well spread and rather more evenly distributed network of collaborations with countries across the world, when compared with its network of collaboration on SARS and MERS. While its strongest collaboration has been with the United States, the names of many other countries appear on its network with no particular country standing out distinctly. Italy, as a country that was highly affected by the Covid19 outbreak, has been exceptionally well represented on this map with a very strong link of collaboration with the United States, followed by United Kingdom at a smaller scale. This pattern of exceptional representation of highly affected countries on the maps has to a lesser extent extended to Iran, Spain, France and Brazil as countries severely affected by the Covid-19 outbreak at early stages of the global spread.

According to the colour coding of the maps, SARS studies in which authors affiliated with the institutes in the Netherlands were involved have, on average, received the highest number of citations. This is followed by studies of authors from Germany. These are two countries whose authors have published considerable number of documents on SARS and received high number of citations at the same time. This pattern was, to some extent, repeated in relation to the MERS literature, with studies from the Netherlands, Germany and United Kingdom having received, on average, the highest number of citations. For studies published on Covid-19, publications from China have so far stood out in terms of both the magnitude of research activities and the average number of citations. 


\section{Discussions}

Outbreaks of infectious diseases have often shown a pattern of generating a quick surge of publications on their respective topics, such that they often create an entirely new literature over a short period of time (Olijnyk 2015; Tian and Zheng 2015). By all measures, however, the influx of the research publications that began to emerge following the 2019 Novel coronavirus outbreak outsizes those of the previous cases in the history of coronaviruses, and perhaps arguably, in the history of infectious diaereses (Tian and Zheng 2015). This has certainly marked a new milestone in the timeline of research on coronaviruses which dates back to 1968 (Almeida et al. 1968). According to the editor-in-chief of the Journal of Virology, as quoted in an article of The Scientist magazine (Jarvis 2020), this surge of research outputs has been to the extent that has inundated established coronavirus researchers and domain experts with peer review requests to an extent that they are unable to cope. Parallel to such intensified efforts on the research, peer review and editorial fronts, widespread efforts have also been underway in synthesising, summarising and visualising these rapid developments, a pattern that has also been observed-though at much smaller scales - with respect to the previous epidemics of viral diseases (Kostoff and Morse 2011). In line with these endeavours, this work also aimed at quantifying and analysing scientometric aspects of the Covid-19 literature in contrast with those of the previous major coronavirus diseases, i.e. SARS and MERS. The focus for sourcing these literatures has been on peer reviewed and published studies.

An analysis of the timeline of the development of publications on coronaviruses made clear that the SARS outbreak constitutes the first major milestone in the history of this research, an event that brought a then-unprecedented amount of attention to this topic. While Scopus has indexed a total of nearly 4400 studies on coronaviruses from 1968 to 2002, the three immediate years post SARS outbreak (i.e. November 2002) have each recorded nearly 1000 coronavirus publications. This means that following the SARS outbreak, the then 36 years old literature of coronaviruses almost expanded by $70 \%$ within only three years. This trend, however, did not persist and did not extend to the years succeeding 2005, as from 2005 onwards, a gradual decline in the rate of publications on coronaviruses began. This continued until the 2012 MERS outbreak, another event that reinvigorated the coronavirus research, though not as substantially as that of the SARS epidemic. The pattern of a few years of increase in research activities on coronaviruses followed by a gradual decline, which had occurred in relation to SARS, also repeated itself in a similar fashion after the MERS outbreak.

The outbreak of 2019 Novel Coronavirus, however, marks a unique milestone in this timeline. The magnitude of scholarly outputs prompted by this novel virus was to the extent that, in less than five months, 12,000 publications was already indexed by Scopus, a number that nearly equals $70 \%$ of the total amount of literature generated on coronaviruses during the 50 years of this research prior to 2020. In fact, since April 2020, we have made five consistent recordings of the number of Scopus-listed publications linked to Covid-19 at five cross-sections of time, the last of which representing our Scopus search on 21 July 2020 as the revision of this article was being drafted. This set of records may give an approximation for the current rate of accumulation of studies on this topic. According to Fig. 17 that visualises these records, the trend seems to be rather linear, although the rate seems to have increased since May 2020. Our very latest Scopus search on 21 July 2020 showed that the number has reached 30,400 items. If the current trend continues, then by the end of 2020, approximately another 50,000 articles could be expected on this topic, 


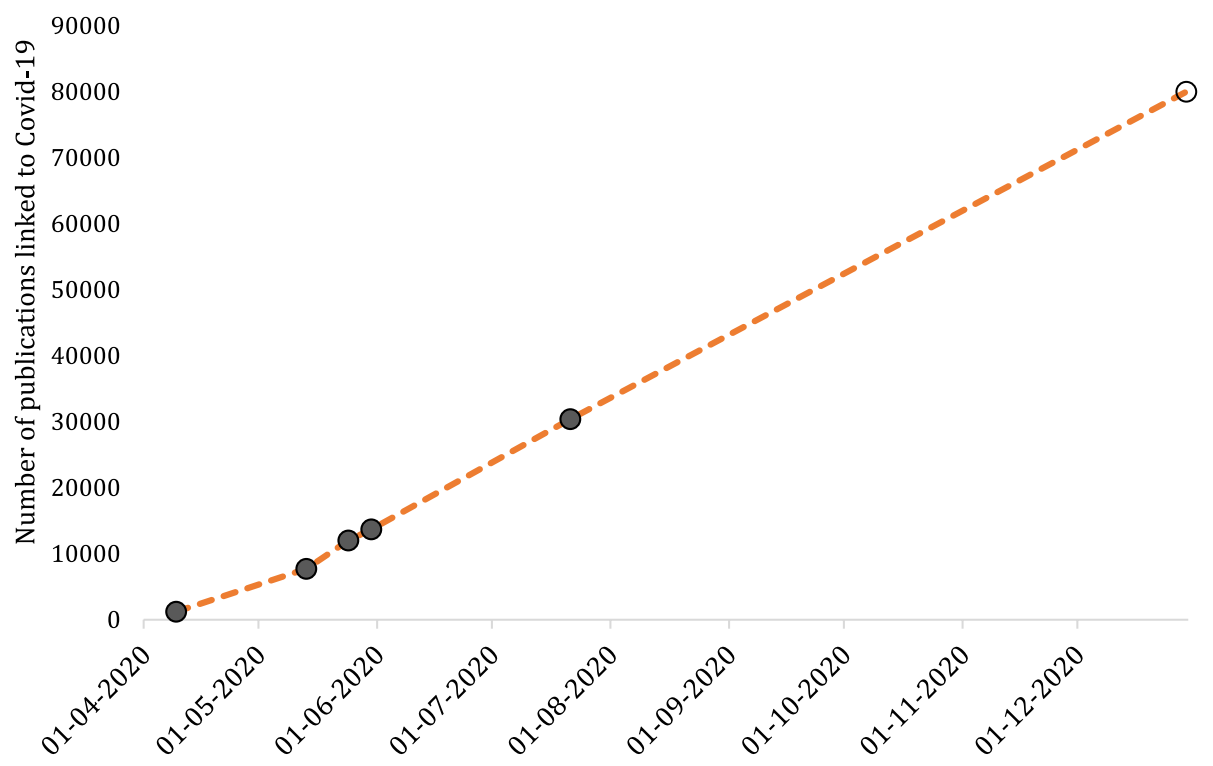

Fig. 17 The number of studies related to Covid-19 indexed by Scopus at five different points in time since April 2020, along with a projection of the number at the end of 2020

making the total size of this literature exceed 80,000 items. A conservative projection could be obtained using the rate of accumulation associated with the time period between April and May 2020, the earlier stage of the emergence of studies on this topic. This would yield an estimate of approximately 70,000 publications by the end of 2020 .

By retrieving and disentangling the literatures linked to these three respiratory diseases, we sought to discover similarities and discrepancies of their research landscape from scientometric perspectives. The most interesting pattern was the recurrence of three distinct clusters of studies in each literature as suggested by keyword co-occurrence analyses. It appeared that, following each outbreak, an early cluster of studies first emerged, addressing issues attributable to the public emergency management aspects of a pandemic, such as prediction of disease propagation, measures of outbreak control, public policy making, and concerns related to the protection of medical professionals and mental health. Accordingly, as a result of these three outbreaks, three significant cohort of studies related to health emergency management have already emerged, which, if synthesised effectively, could constitute a valuable and evidence-based guide to help government and communities better prepare for and react to possible future disease outbreaks, at least of those of the respiratory nature.

Citation and bibliographic coupling analysis at the level of journals demonstrated that firstly, Covid-19 studies are scattered across a broader variety of sources and subject categories, and secondly, its network of journal relations is still not as cohesive as those of the SARS and MERS literatures. As the literature on Covid-19 further develops, more cohesive patterns of bibliographic coupling or journal citations may form. While Journal of Virology and Emerging Infectious Diseases seem to have been two major outlets commonly prominent across both SARS and MERS literatures, their presence in the literature of Covid-19 seem to not be distinctly notable. Instead, a great portion of Covid-19 studies have concentrated across three journals: The Lancet, The BMJ, 
and Journal of Medical Virology. While major multidisciplinary journals, particularly Science, Nature, PNAS and PLOS ONE, have, to varying degrees, been active in publishing studies on all three topics, their influence is most notable in relation to the SARS literature where they have published a substantial portion of studies. Those studies happen to have been recipients of relatively high number of citations.

The involvement of authors from various countries on the publications linked to these three diseases seem to be distinctly correlated with the regions where the outbreaks originated, with authors from China, for example, being much more strongly represented on SARS and Covid-19 studies, two diseases whose origin of outbreaks were in that country. Middle Eastern countries, on the other hand, are exceptionally represented in the MERS literature.

The questions of where the Covid-19 literature is headed, how big it will grow in the next coming months/years, at what point in time the rate of publications on this topic is going to slow down and how widely this literature is going to spread across journals and subject categories are just a few examples of questions that will only be determined by time. These scholarly patterns may also be influenced by future success or failure of countries in controlling the extent of the surge and re-surge of outbreaks and by possible medical discoveries in relation to vaccine and treatment. But given the pace at which scholarly outputs are currently emerging, the number of under-review preprints, the extent of studies and clinical trials that have already been conceived around the world; and also considering the seemingly long-lasting and far-reaching consequences of this global emergency that have impacted on aspects of life, it will probably not be so soon before we observe a decline in Covid-19 research interest.

\section{Summary and conclusions}

Macro-syntheses of the scholarly literatures generated in response to the three most significant outbreaks of coronaviruses, i.e. SARS, MERS and Covid-19, revealed interesting structural similarities across all three literatures and suggested that they are commonly made up of three major segments. In each case, publications related to public health emergency management have been the earliest to merge, followed by studies on virus chemistry and clinical treatments. However, despite this similarity of the structures and the recurring patterns of publications following each outbreak, the magnitude of the rate of scholarly research prompted by the 2019 Novel Coronavirus outbreak remains a striking phenomenon, arguably, unique in the history of scientific publication. The main motive of this study was to document this major milestone and the magnitude of these scientific efforts. It is, per se, a heartening observation to note how the 2020 global health crisis has mobilised the efforts of scholars across the globe and across scientific disciplines. A question that naturally follows, however, would be to discover how the rush to combat this global crisis through scientific work has influenced the rigour and quality of the publications. This could warrant further research on this rapidly evolving literature in the form of quality assessment of publications.

\section{Appendix}

See Figs. , 18, 19, 20, 21, 22, 23, 24, 25, 26, 27, 28, 29, 30, and 31. 


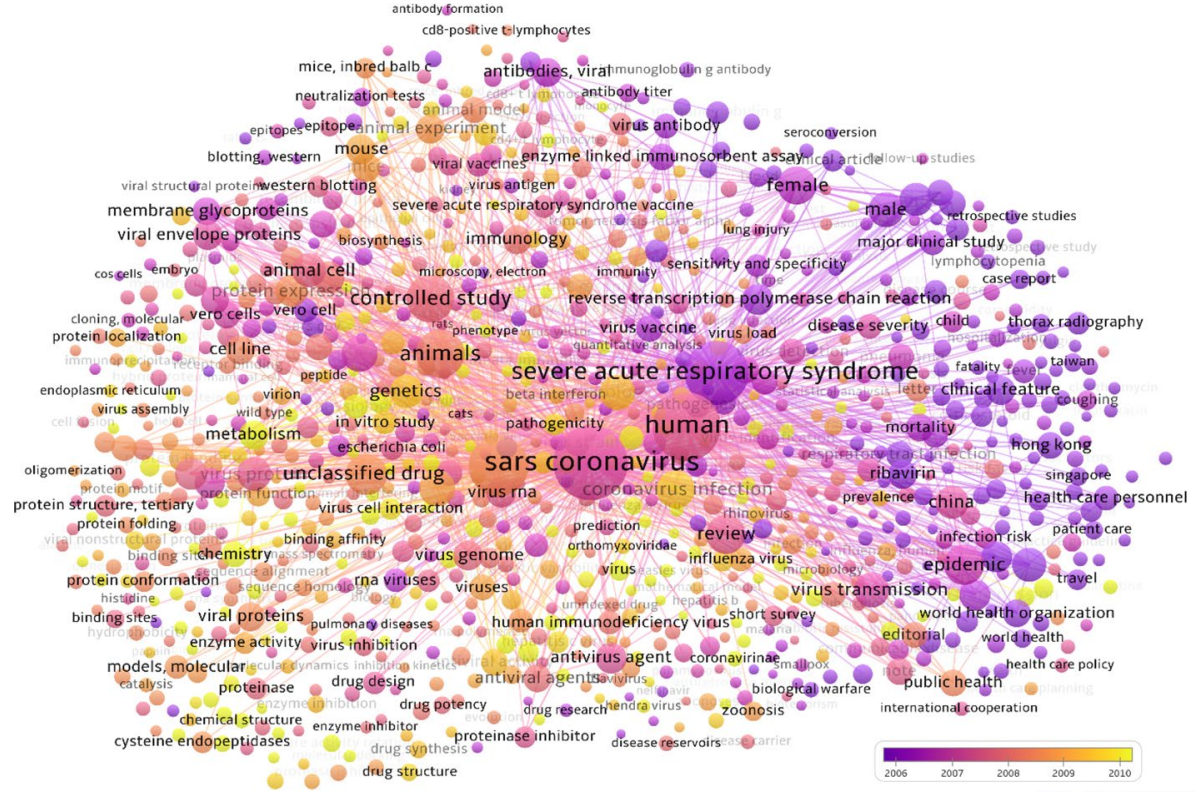

Fig. 18 Map of SARS keyword co-occurrence overlaid with the average publication year

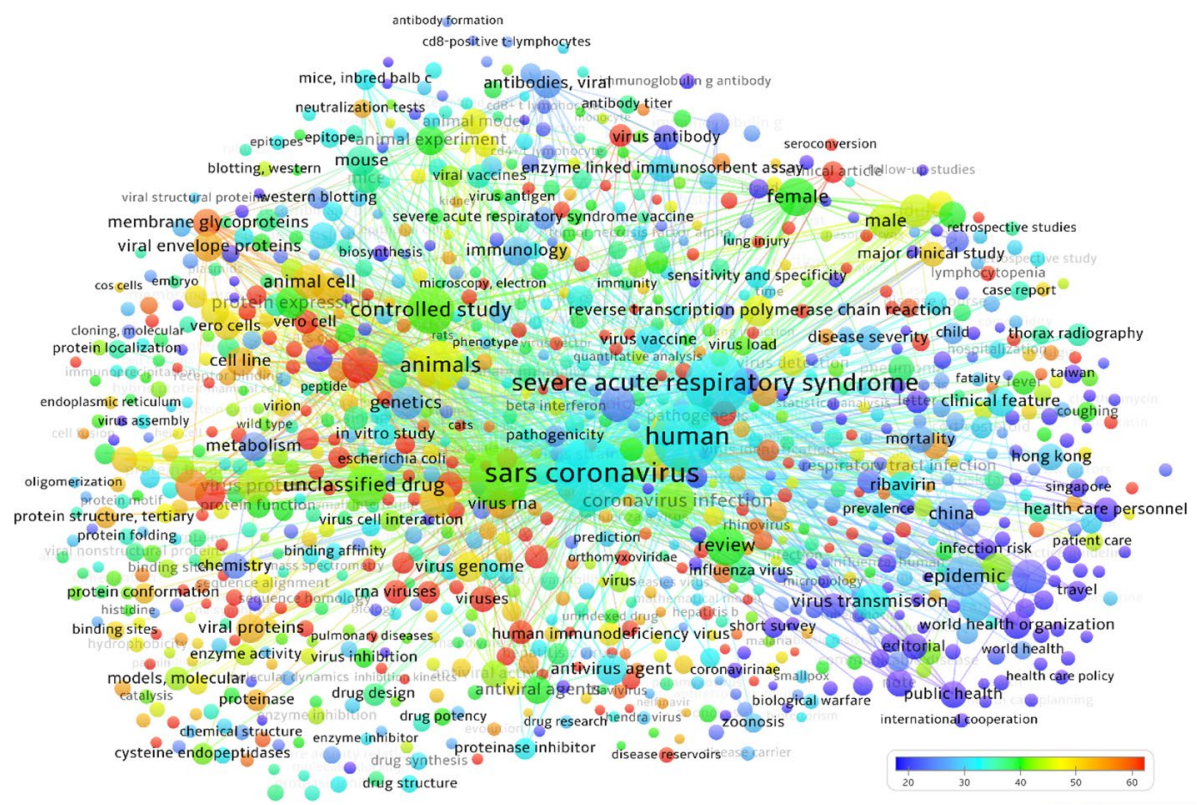

Fig. 19 Map of SARS keyword co-occurrence overlaid with the average citation number 


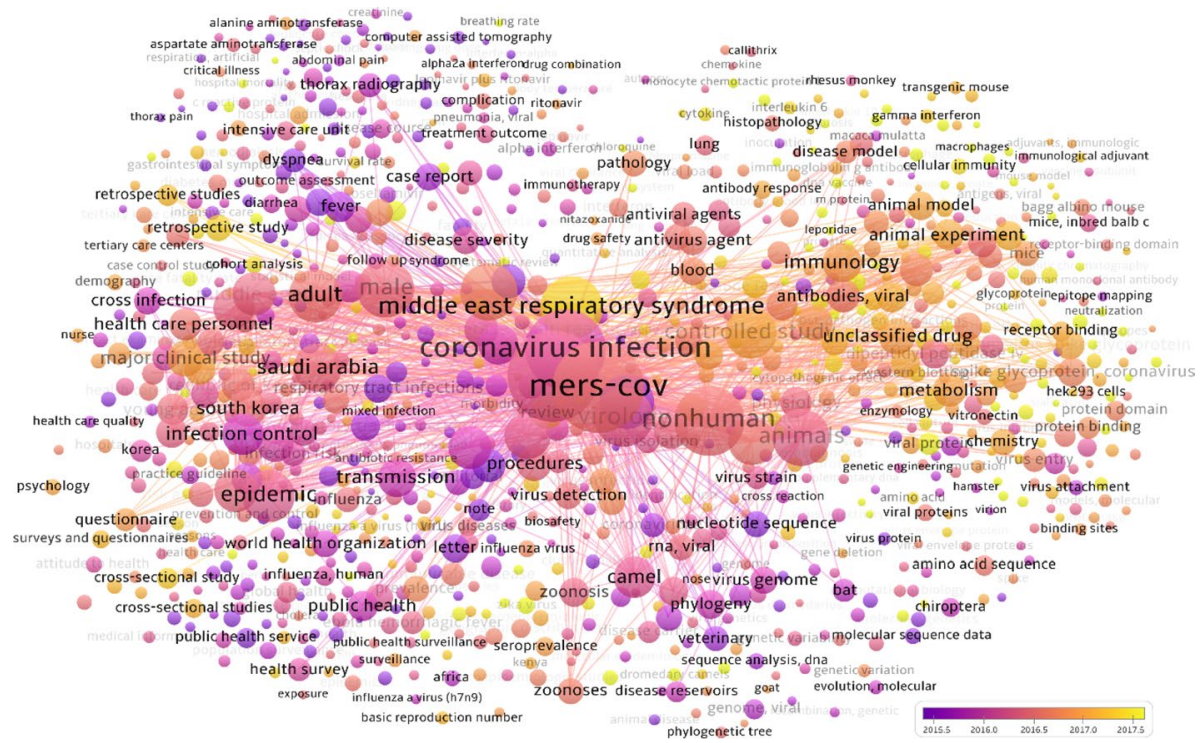

Fig. 20 The map of keyword co-occurrence for MERS overlaid with the colour-coding of the average year of publication

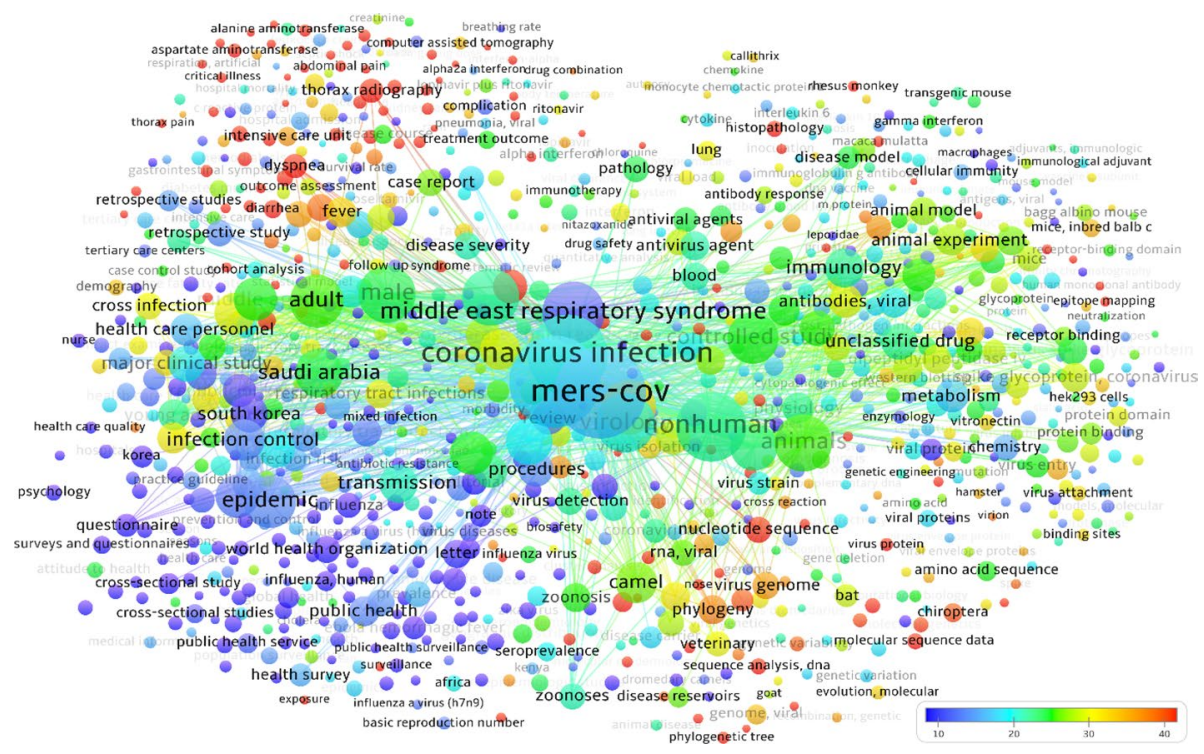

Fig. 21 The map of keyword co-occurrence for MERS overlaid with the colour-coding of the average citation number 


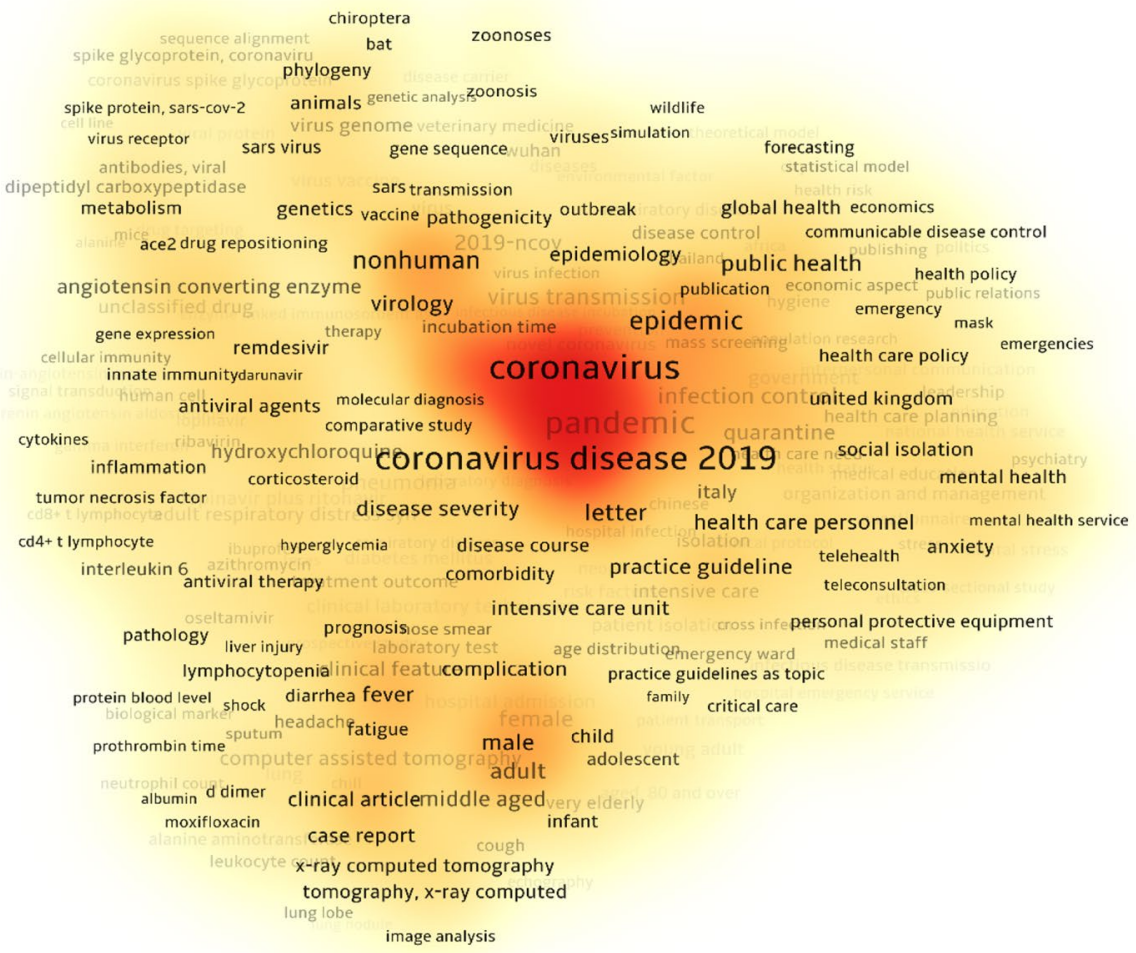

Fig. 22 The heatmap of keyword co-occurrence for Covid-19 
chiroptera
spike glygoprotein, coronaving
phylogeny bat dipeptidyl earboxypeptidase sarstransmission
metabolism - acezdrug repositioning nonhuman epidemiology public health healthpolicy angiotensin converting enzyme publication econonig aspechealth policy gene expression virology a remdesivir therapy incubation time epidemic mask gene expression remdesivir coronaviruS health care policy
innate immunitydarunavir - antiviral agents molecular diagnosis ytokines comparative study pandemic gurantinge social isolation inflammation corticosteroid Coronavirus disease 2019 social isolation mental health - 0 disease severity letter health care personnel omental health service

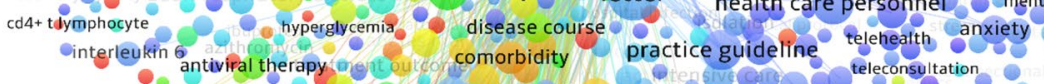

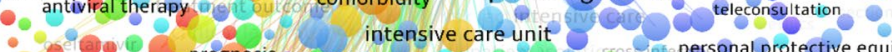

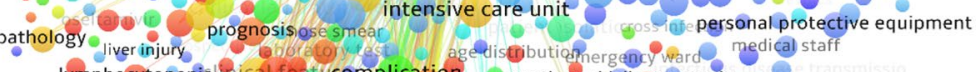
Iymphocytopenial hicalffeaticomplication practice guidelines as topic

protein bloodfevel shock diarrhea fever prothrombir time splem male child - e adult

albumin dimer clinical articlemiddle aged moxifloxacin case report infant

- ecteray computed tomography tomography, $x$-raycomputed lunglobe

- image analysis

Fig. 23 The map of keyword co-occurrence for Covid-19 overlaid with the colour-coding the average citation number

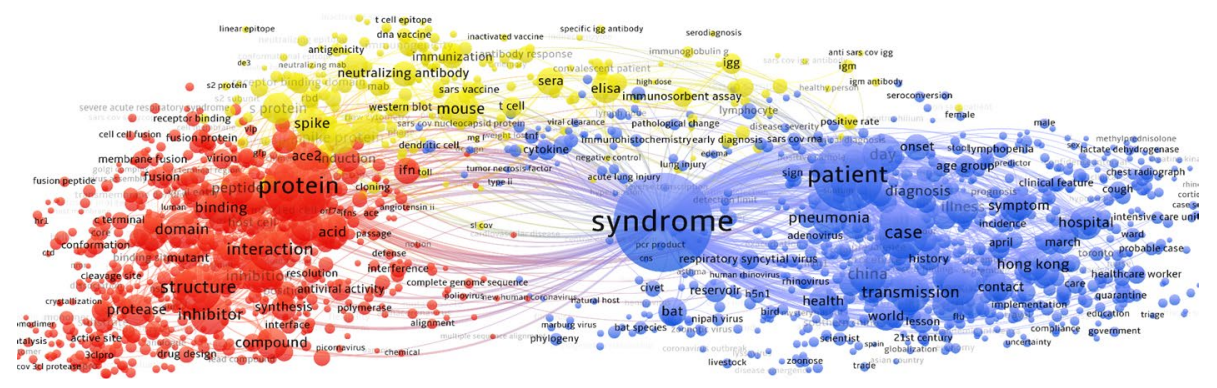

Fig. 24 The map of term co-occurrence for SARS based on text analysis of titles and abstracts 


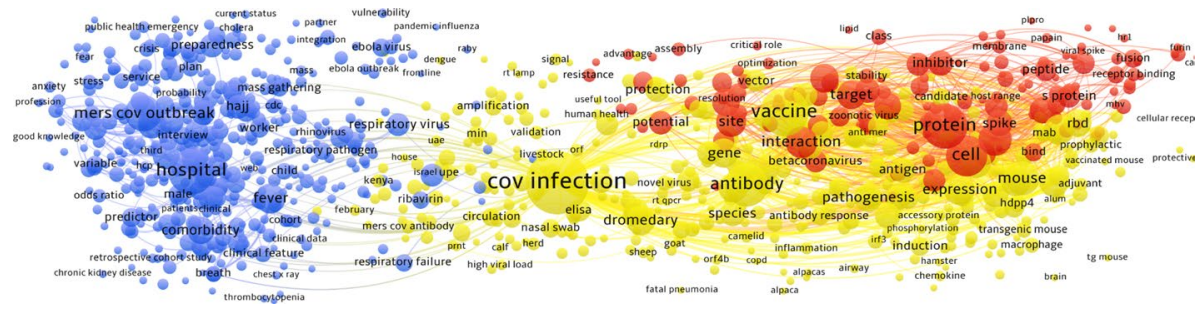

Fig. 25 The map of term co-occurrence for MERS based on text analysis of titles and abstract

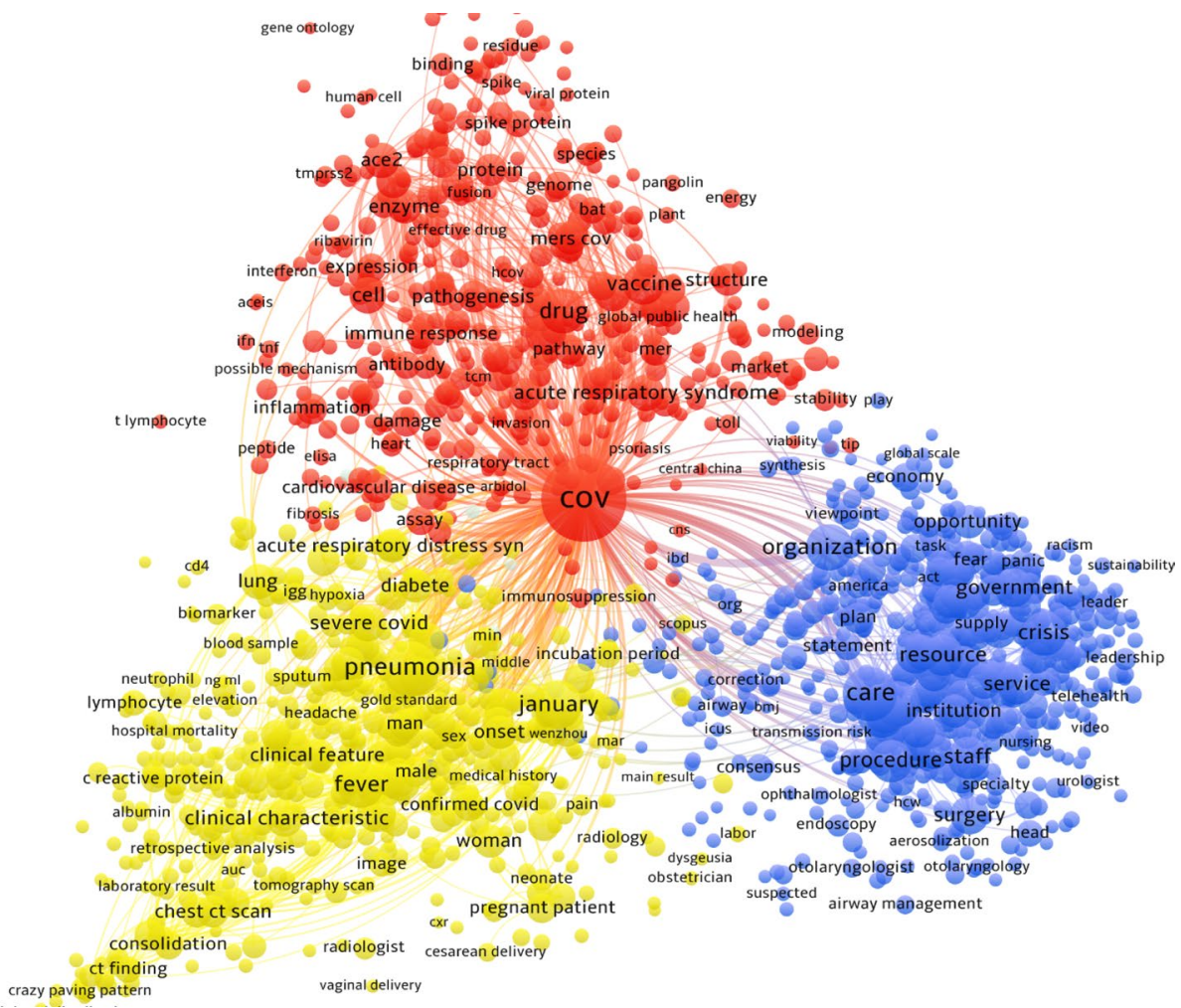

Fig. 26 The map of term co-occurrence for MERS based on text analysis of titles and abstracts 


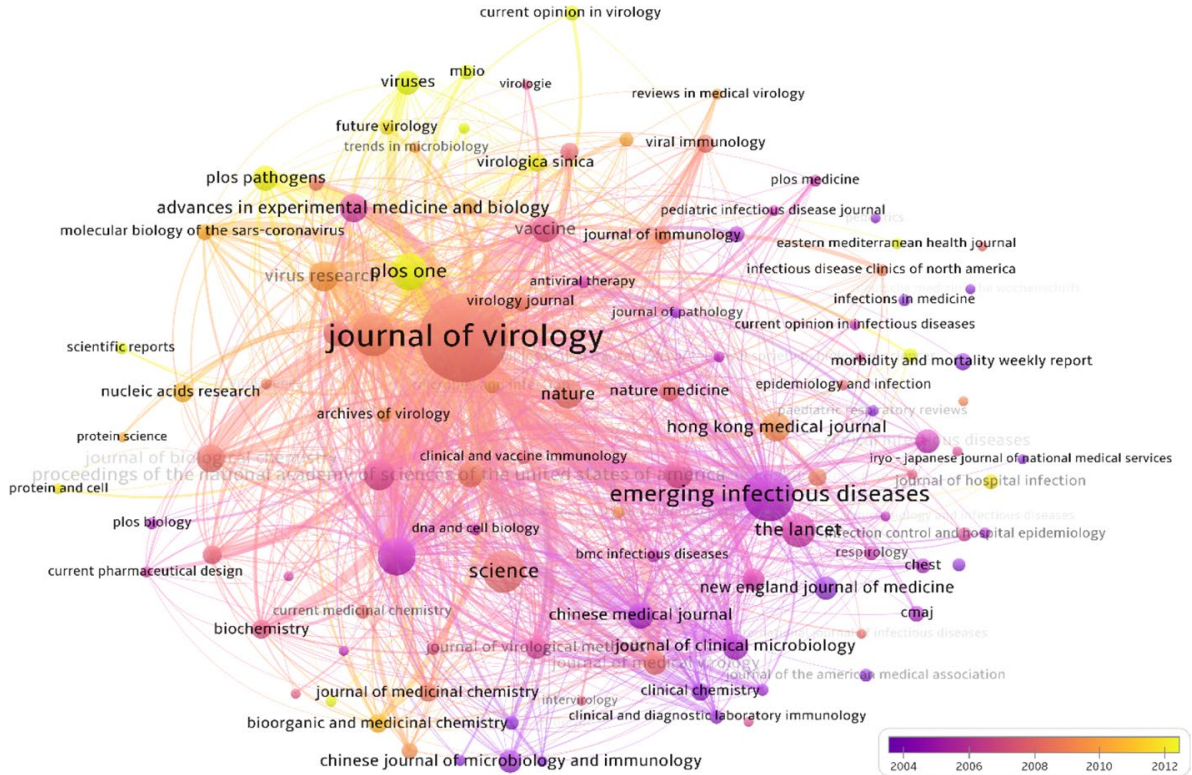

Fig. 27 The map of bibliographic coupling of sources for SARS overlaid with the colour-coding of the average publication year

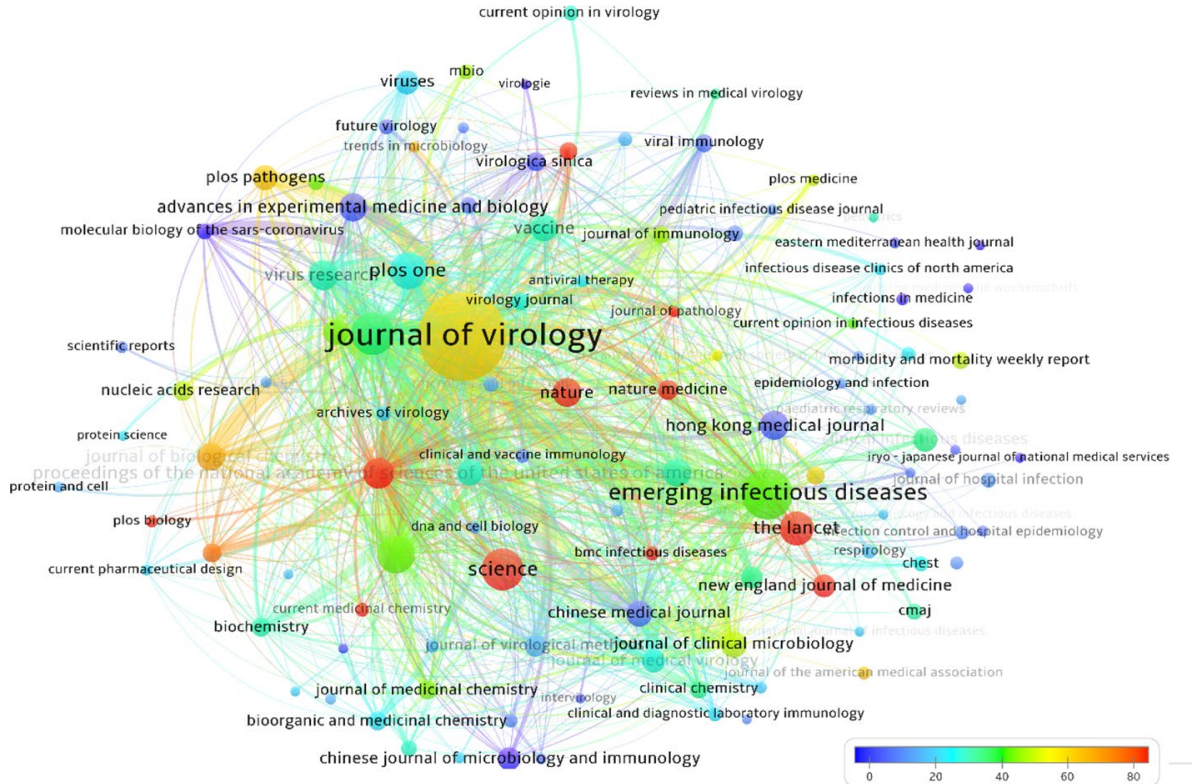

Fig. 28 The map of bibliographic coupling of sources for SARS overlaid with the colour-coding of the average citation per document 


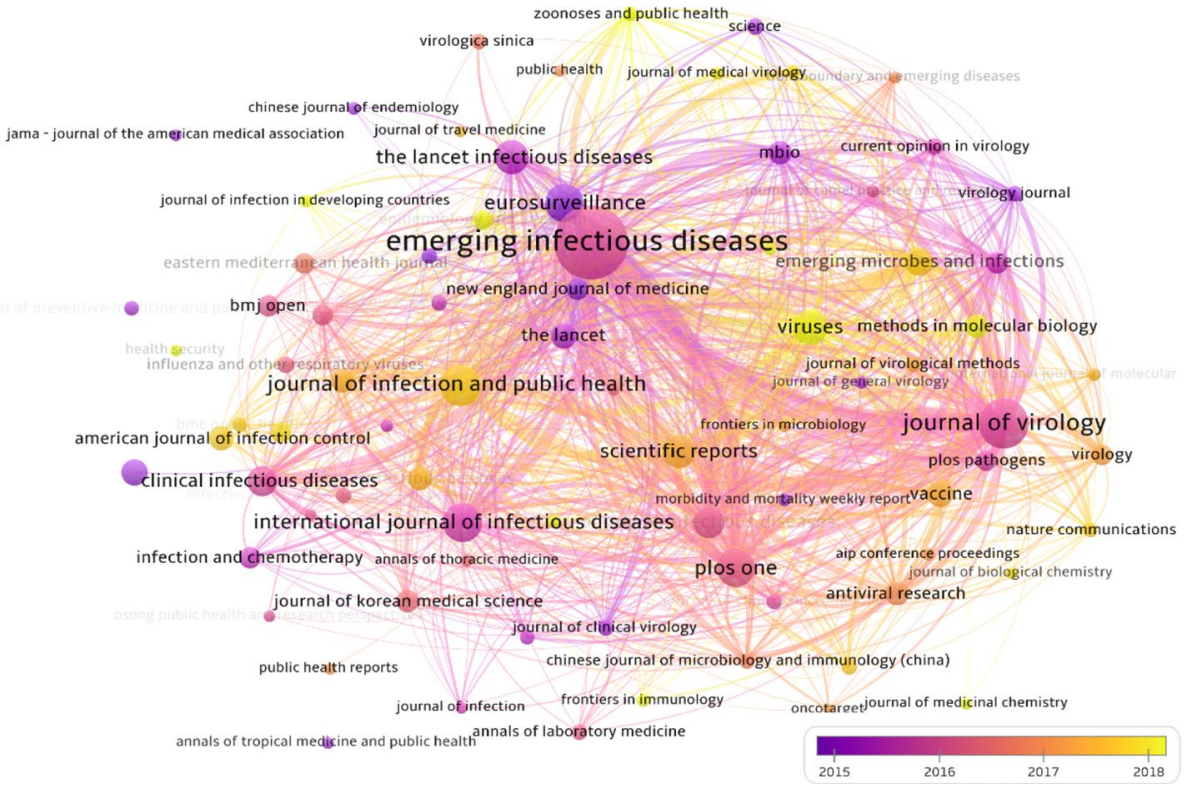

Fig. 29 The map of bibliographic coupling of sources for MERS overlaid with the colour-coding of the average publication year

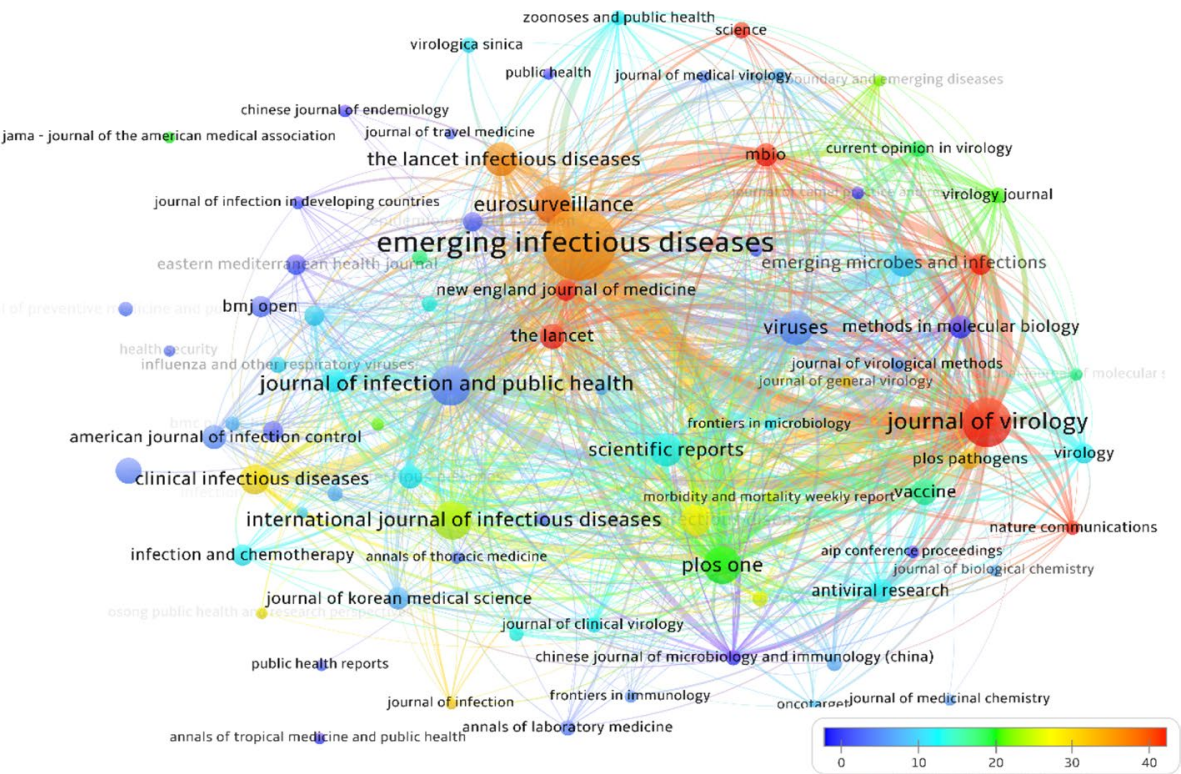

Fig. 30 The map of bibliographic coupling of sources for MERS overlaid with the colour-coding of the average citation per document 


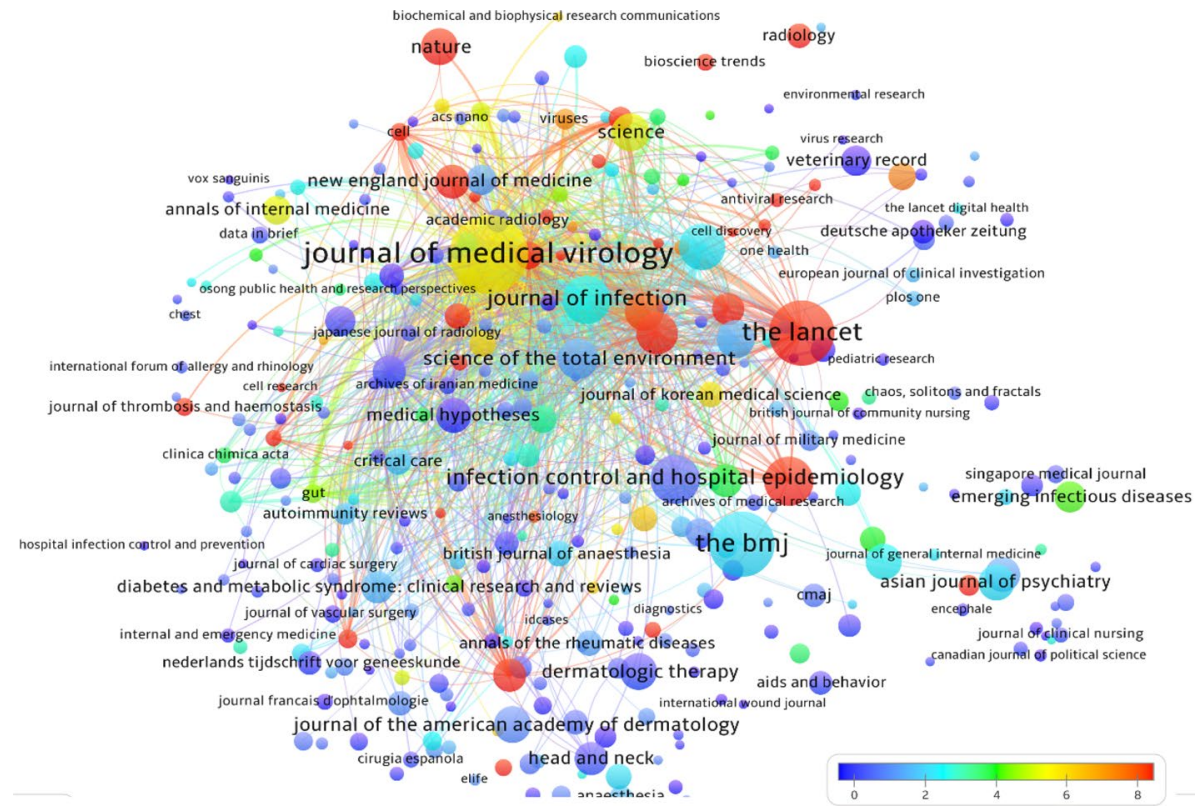

Fig. 31 The map of bibliographic coupling of sources for Covid-19 overlaid with the colour-coding of the average citation per document

\section{References}

Almeida, J., Berry, D., Cunningham, C., Hamre, D., Hofstad, M., Mallucci, L., et al. (1968). Coronaviruses. Nature, 220(650), 2.

Anggraeni, S., Maulidina, A., Dewi, M. W., Rahmadianti, S., Rizky, Y. P. C., Arinalhaq, Z. F., et al. (2020). The deployment of drones in sending drugs and patient blood samples covid-19. Indonesian Journal of Science and Technology, 5(2), 18-25.

Barmparis, G. D., \& Tsironis, G. P. (2020). Estimating the infection horizon of covid-19 in eight countries with a data-driven approach. Chaos Solitons and Fractals. https://doi.org/10.1016/j.chaos.2020.10984 2 .

Bekiros, S., \& Kouloumpou, D. (2020). Sbdiem: A new mathematical model of infectious disease dynamics. Chaos Solitons and Fractals. https://doi.org/10.1016/j.chaos.2020.109828.

Boccaletti, S., Ditto, W., Mindlin, G., \& Atangana, A. (2020). Modeling and forecasting of epidemic spreading: The case of covid-19 and beyond. Chaos Solitons and Fractals. https://doi.org/10.1016/j. chaos.2020.109794.

Bonilla-Aldana, D. K., Quintero-Rada, K., Montoya-Posada, J. P., Ramírez-Ocampo, S., Paniz-Mondolfi, A., Rabaan, A. A., et al. (2020). Sars-cov, mers-cov and now the 2019-novel cov: Have we investigated enough about coronaviruses?-A bibliometric analysis. Travel medicine and infectious disease, 33, 101566.

Brainard, J. (2020). Scientists are drowning in covid-19 papers Can new tools keep them afloat? Science. https://doi.org/10.1126/science.abc7839.

Bychkova, O. V. (2020). Covid-19 and climate change reactions: Sts potential of online research. Social Anthropology. https://doi.org/10.1111/1469-8676.12884.

Cavanagh, D. (2005). Coronaviridae: A review of coronaviruses and toroviruses. In A. Schmidt, et al. (Eds.), Coronaviruses with special emphasis on first insights concerning sars (pp. 1-54). New york: Springer.

Chahrour, M., Assi, S., Bejjani, M., Nasrallah, A. A., Salhab, H., Fares, M., et al. (2020). A bibliometric analysis of covid-19 research activity: A call for increased output. Cureus. https://doi.org/10.7759/ cureus. 7357. 
Chang, L., Yan, Y., \& Wang, L. (2020). Coronavirus disease 2019: Coronaviruses and blood safety. Transfusion Medicine Reviews. https://doi.org/10.1016/j.tmrv.2020.02.003.

Chen, Y., Liu, Q., \& Guo, D. (2020). Emerging coronaviruses: Genome structure, replication, and pathogenesis. Journal of medical virology, 92(4), 418-423.

Cherry, J. D., \& Krogstad, P. (2004). Sars: The first pandemic of the 21st century. Pediatric research, 56(1), 1-5.

Colavizza, G., Costas, R., Traag, V. A., Van Eck, N. J., Van Leeuwen, T., \& Waltman, L. (2020). A scientometric overview of cord-19. BioRxiv, 3, 993.

Cortegiani, A., Ingoglia, G., Ippolito, M., Giarratano, A., \& Einav, S. (2020). A systematic review on the efficacy and safety of chloroquine for the treatment of covid-19. Journal of Critical Care. https://doi. org/10.1016/j.jcrc.2020.03.005.

Dandekar, A., \& Ghai, R. (2020). Migration and reverse migration in the age of covid-19. Economic and Political Weekly, 55(19), 28-31.

De Vos, J. (2020). The effect of covid-19 and subsequent social distancing on travel behavior. Transportation Research Interdisciplinary Perspectives. https://doi.org/10.1016/j.trip.2020.100121.

Dehghanbanadaki, H., Seif, F., Vahidi, Y., Razi, F., Hashemi, E., Khoshmirsafa, M., et al. (2020). Bibliometric analysis of global scientific research on coronavirus (covid-19). Medical Journal of The Islamic Republic of Iran (MJIRI), 34(1), 354-362.

Golinelli, D., Nuzzolese, A. G., Boetto, E., Rallo, F., Greco, M., Toscano, F., et al. (2020). The impact of early scientific literature in response to covid-19: A scientometric perspective. MedRxiv. https://doi. org/10.1101/2020.04.15.20066183.

Haghani, M., Bliemer, M. C. J., Goerlandt, F., \& Li, J. (2020). The scientific literature on coronaviruses, covid-19 and its associated safety-related research dimensions: A scientometric analysis and scoping review. Safety Science, 129, 104806.

Hossain, M. M. (2020). Current status of global research on novel coronavirus disease (covid-19): A bibliometric analysis and knowledge mapping. Available at SSRN Journal. https://doi.org/10.2139/ ssrn.3547824.

Jarvis, C. (2020). Journals, peer reviewers cope with surge in covid-19 publications. Solana beach: The Scientist.

Kagan, D., Moran-Gilad, J., \& Fire, M. (2020). Scientometric trends for coronaviruses and other emerging viral infections. BioRxiv, 46, 416.

Karaseva, A. (2020). The legal void and covid-19 governance. Social Anthropology. https://doi. org/10.1111/1469-8676.12825.

Knight, C. A. (1954). The chemical constitution of viruses. In K. M. Smith \& M. A. Lauffer (Eds.), Advances in virus research (pp. 153-182). Waltham: Academic Press.

Kostoff, R. N., \& Morse, S. A. (2011). Structure and infrastructure of infectious agent research literature: Sars. Scientometrics, 86(1), 195-209.

Kumar, K. (2020). Author productivity of covid-19 research output globally: Testing lotka's law. Available at SSRN Journal. https://doi.org/10.2139/ssrn.3603889.

Le Bras, P., Gharavi, A., Robb, D.A., Vidal, A.F., Padilla, S., Chantler, M.J., 2020. Visualising covid-19 research. arXiv, arXiv: 2005.06380.

Lee, D., \& Lee, J. (2020). Testing on the move: South korea's rapid response to the covid-19 pandemic. Transportation Research Interdisciplinary Perspectives. https://doi.org/10.1016/j.trip.2020.100111.

Lim, Y. X., Ng, Y. L., Tam, J. P., \& Liu, D. X. (2016). Human coronaviruses: A review of virus-host interactions. Diseases, 4(3), 26.

Mcintosh, K., (1974). Coronaviruses comparative review. In W.Arber et al (Eds.), Current topics in microbiology and immunology ergebnisse der mikrobiologie und immunitätsforschung (pp.85-129). Newyork: Springer.

Mulchandani, P. (2020). Covid-19 crisis: Economic stimulus packages and environmental sustainability. Economic and Political Weekly. https://doi.org/10.2139/ssrn.3663113.

Myint, S. (1994). Human coronaviruses: A brief review. Reviews in Medical Virology, 4(1), 35-46.

Ndaïrou, F., Area, I., Nieto, J. J., \& Torres, D. F. M. (2020). Mathematical modeling of covid-19 transmission dynamics with a case study of wuhan. Chaos Solitons and Fractals. https://doi.org/10.1016/j. chaos.2020.109846.

Olijnyk, N. V. (2015). An algorithmic historiography of the ebola research specialty: Mapping the science behind ebola. Scientometrics, 105(1), 623-643.

Postnikov, E. B. (2020). Estimation of covid-19 dynamics "on a back-of-envelope": Does the simplest sir model provide quantitative parameters and predictions? Chaos Solitons and Fractals. https://oi. org/10.1016/j.chaos.2020.109841. 
Reardon, T., Mishra, A., Nuthalapati, C. S. R., Bellemare, M. F., \& Zilberman, D. (2020). Covid-19's disruption of india's transformed food supply chains. Economic and Political Weekly, 55(18), 18-22.

Ribeiro, M. H. D. M., Da Silva, R. G., Mariani, V. C., \& Coelho, L. D. S. (2020). Short-term forecasting covid-19 cumulative confirmed cases: Perspectives for brazil. Chaos Solitons and Fractals. https://oi. org/10.1016/j.chaos.2020.109853.

Sedaju, A., Haryono, S., \& Anisahwati, N. (2020). Flexible work arrangement in manufacturing during the covid-19 pandemic: An evidence-based study of indonesian employees. International Journal of Advanced Science and Technology, 29(6), 3914-3924.

Sohrabi, C., Alsafi, Z., \& O'neill, N., Khan, M., Kerwan, A., Al-Jabir, A., Iosifidis, C., Agha, R.,. (2020). World health organization declares global emergency: A review of the 2019 novel coronavirus (covid19). International Journal of Surgery, 76, 71-76.

Tian, D., \& Zheng, T. (2015). Emerging infectious disease: Trends in the literature on sars and h7n9 influenza. Scientometrics, 105(1), 485-495.

Torres-Salinas, D., Robinson-Garcia, N., \& Castillo-Valdivieso, P. A. (2020). Open access and altmetrics in the pandemic age forescast analysis on covid-19 related literature. BioRxiv, 26, 420.

Van Eck, N., \& Waltman, L. (2010). Software survey: Vosviewer, a computer program for bibliometric mapping. Scientometrics. https://doi.org/10.1007/s11192-009-0146-3.

Wang, L., Wang, Y., Ye, D., \& Liu, Q. (2020). Review of the 2019 novel coronavirus (sars-cov-2) based on current evidence. International Journal of Antimicrobial Agents. https://doi.org/10.1016/j.ijantimica g.2020.105948.

Wesemann, C., Pieralli, S., Fretwurst, T., Nold, J., Nelson, K., Schmelzeisen, R., et al. (2020). 3-d printed protective equipment during covid-19 pandemic. Materials. https://doi.org/10.3390/ma13081997.

Zhang, X., Ma, R., \& Wang, L. (2020). Predicting turning point, duration and attack rate of covid-19 outbreaks in major western countries. Chaos, Solitons and Fractals.. https://doi.org/10.1016/j.chaos .2020 .109829

\section{Affiliations}

\section{Milad Haghani ${ }^{1} \cdot$ Michiel C. J. Bliemer ${ }^{1}$}

Milad Haghani

Milad.haghani@sydney.edu.au

1 Institute of Transport and Logistics Studies, The University of Sydney Business School, The University of Sydney, Sydney, NSW, Australia 